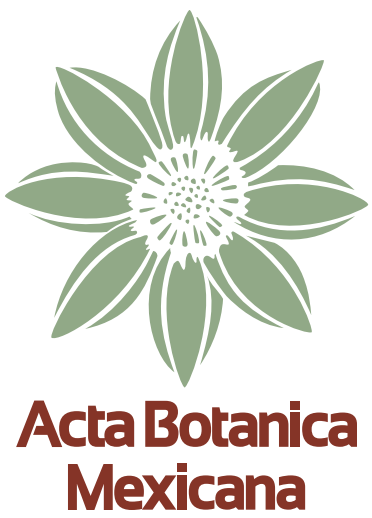

\title{
Aspectos ecológicos, taxonómicos y de distribución de cianobacterias bentónicas en cinco ríos de la región central de México
}

\author{
Ecological, taxonomic and distributional aspects \\ of benthic cyanobacteria in five streams of central \\ Mexico
}

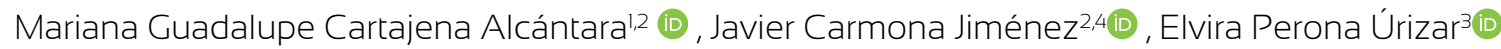

\section{Resumen:}

Antecedentes y Objetivos: Las cianobacterias son algas procariontes oxifotótrofas presentes en gran variedad de hábitats y son componentes importantes de las comunidades bentónicas en muchos ambientes continentales y marinos. En México su diversidad en ríos es poco conocida; por ello, este estudio contribuye al conocimiento ficoflorístico en cinco ríos de la región central de México. Se incluyen descripciones e ilustraciones, estimaciones de su diversidad, un análisis de las relaciones entre formas de crecimiento y factores microambientales, y se discute la validez de su caracterización morfológica y ecológica en la delimitación de las especies encontradas.

Métodos: Se realizaron muestreos en secas y lluvias en dos ríos silíceos y tres ríos calcáreos del centro de México. Se registró la temperatura del agua, conductividad específica y $\mathrm{pH}$, se analizaron iones mayores $\left(\mathrm{Ca}^{2+}, \mathrm{Mg}^{2+}, \mathrm{K}^{+}, \mathrm{Na}^{+}, \mathrm{Si}-\mathrm{SiO}_{2}, \mathrm{HCO}_{3^{-}}, \mathrm{Cl}^{-}, \mathrm{SO}_{4}{ }^{3-}\right)$ y nutrientes $\left(\mathrm{NH}_{4}^{+}, \mathrm{NO}_{2^{-}}, \mathrm{NO}_{3}{ }^{2-}, \mathrm{PO}_{4}{ }^{3-}\right)$, se estimó la diversidad de las cianobacterias registradas con el índice de Shannon-Weaver y se describieron sus hábitats y microhábitats. La identificación taxonómica se realizó utilizando literatura especializada.

Resultados clave: Se identificaron cuatro asociaciones y nueve especies pertenecientes a tres órdenes, siete familias y siete géneros. Se distinguieron dos grupos: el formado por Nostoc parmelioides-Coleodesmium wrangelii y Phormidium lividum-Leptolyngbya truncata en ríos silíceos de montaña y el compuesto por Schizothrix mexicana-Phormidium subfuscum, Homoeothrix juliana-Heteroleibleinia fontana y Nostoc verrucosum en ríos calcáreos cálidos. Se reconocieron dos patrones de distribución ecológica: 1) las especies del orden Nostocales presentes en condiciones estresantes de luz y velocidad de corriente y 2) los taxa de los órdenes Oscillatoriales y Synechococcales registrados en sitios con valores promedio de luz y flujo de agua. Conclusiones: La caracterización morfológica y ecológica de especies de cianobacterias contribuye a su conocimiento ficoflorístico y es la base para futuras investigaciones sobre taxonomía, sistemática y uso sustentable de recursos algales.

Palabras clave: asociaciones, diversidad, ríos calcáreos, ríos silíceos.

\section{Abstract:}

Background and Aims: Cyanobacteria are oxyphototrophic prokariotic algae present in great variety of habitats and they are important components of the benthic communities in many continental and marine environments. In Mexico, their diversity in streams is less known; therefore, this study contributes to phycofloristic knowledge in five streams of the central region of Mexico. Descriptions and illustrations, estimations of their diversity, an analysis of the relationships between growth forms and microenvironmental factors are included, and the validity of their morphological and ecological characterization in the delimitation of the species found is discussed.

Methods: Sampling was carried out in dry and rainy seasons in two siliceous streams and three calcareous streams of central Mexico. Water temperature, specific conductivity and $\mathrm{pH}$ were recorded, major ions $\left(\mathrm{Ca}^{2+}, \mathrm{Mg}^{2+}, \mathrm{K}^{+}, \mathrm{Na}^{+}, \mathrm{Si}-\mathrm{SiO}_{2}, \mathrm{HCO}_{3}^{-}, \mathrm{Cl}^{-}, \mathrm{SO}_{4}{ }^{3-}\right)$ and nutrients $\left(\mathrm{NH}_{4}^{+}, \mathrm{NO}_{2}^{-}, \mathrm{NO}_{3}{ }^{2-}, \mathrm{PO}_{4}{ }^{3-}\right)$ were analyzed, the diversity was estimated of the cyanobacteria registered with the Shannon-Weaver index, and their habitats and microhabitats were described. Taxonomic identification was carried out with specialized literature.

Key results: Four associations and nine species belonging to three orders, seven families and seven genera were identified. Two groups were distinguished: one formed by Nostoc parmelioides-Coleodesmium wrangelii, Phormidium lividum-Leptolyngbya truncata in siliceous and mountain streams, and one composed by Schizothrix mexicana-Phormidium subfuscum, Homoeothrix juliana-Heteroleibleinia fontana and Nostoc verrucosum in calcareous, warm streams. Two ecological distribution patterns were recognized: 1) the Nostocales species present in stressful conditions of light and current velocity and 2) the taxa of Oscillatoriales and Synechoccales registered in sites with mean values of light and water flow.

Conclusions: Morphological and ecological characterization of cyanobacterial species contributes to their phycofloristic knowledge and the base for future research on taxonomy, systematics and sustainable use of algal resources.

Key words: associations, calcareous streams, diversity, siliceous streams.

${ }^{1}$ Universidad Nacional Autónoma de México, Posgrado en Ciencias del Mar y Limnología, Av. Universidad 3000, Circuito exterior s.n., Alcaldía Coyoacán, 04510 Ciudad Universitaria, Cd. Mx., México.

2Universidad Nacional Autónoma de México, Facultad de Ciencias, Laboratorio de Ecosistemas de Ribera, Av. Universidad 3000, Circuito Exterior s.n., Alcaldía Coyoacán, 04510 Ciudad Universitaria, Cd. Mx., México. ${ }^{3}$ Universidad Autónoma de Madrid, Facultad de Ciencias, Departamento de Biología, Edificio de Biología, Calle Darwin 3, Despacho B002, Cantoblanco, España. ${ }^{4}$ Autor para la correspondencia: jcj@ciencias.unam.mx
Recibido: 20 de noviembre de 2019. Revisado: 16 de enero de 2020.

Aceptado por Marie-Stéphanie Samain: 13 de abril de 2020.

Publicado primero en línea: 13 de mayo de 2020.

Publicado: Acta Botanica Mexicana 127(2020).

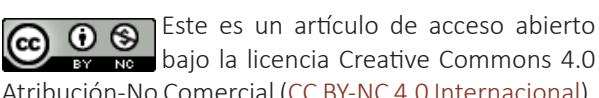

Citar como: Cartajena Alcántara, M. G., J. Carmona Jiménez y E. Perona Úrizar. 2020. Aspectos ecológicos, taxonómicos y de distribución de cianobacterias bentónicas en cinco ríos de la región central de México. Acta Botanica Mexicana 127: e1639. DOI: 10.21829/ abm127.2020.1639

e-ISSN: $2448-7589$ 


\section{Introducción}

Las cianobacterias son algas procariontes oxifototróficas clasificadas en el reino Eubacteria, phyllum Cyanobacteria, clase Cianophyceae y pertenecientes a ocho órdenes (Komárek et al., 2014). Sus especies son comunes en ambientes continentales como humedales, lagos y ríos (Scott y Marcarelli, 2012). En la región central de México existen más estudios sobre cianobacterias de lagos (Ortega Murillo et al., 2015; Betancourt, 2018; Segura, 2018; Trejo, 2018) y de monumentos históricos (Ramírez et al., 2011; Loyo, 2015; Ibarra, 2017), que los que hay acerca de cianobacterias de ríos, donde el conocimiento de su diversidad es menor. En los ríos de la región central de México, los estudios de mayor relevancia han dado a conocer 69 taxa de cianobacterias (Valadez-Cruz et al., 1996; Carmona et al., 2005; Montejano et al., 2005, 2017, 2018; Bojorge-García et al., 2010; Becerra-Absalón et al., 2013, 2018; Rodarte et al., 2014; Maceda et al., 2017; Rodríguez-Flores y Carmona, 2018).

Estudios realizados en ríos han registrado a las cianobacterias como especies importantes, pues son comunes o dominantes en las comunidades de macroalgas (Sheath y Cole, 1992; Montejano et al., 2000; Branco et al., 2001; Zanini et al., 2009; Tonetto et al., 2015). Por ejemplo, Blennothrix ganeshii Watanabe \& Komárek y Placoma regularis Broady \& Ingerfeld muestran amplia distribución en las cuencas del río Pánuco y de México, respectivamente (Carmona et al., 2005; Rodríguez-Flores y Carmona, 2018).

La distribución de cianobacterias bentónicas se ha correlacionado con la conductividad específica y la concentración de nutrientes (Sheath y Cole, 1992; Perona et al., 1998; Branco et al., 2001; Douterelo et al., 2004). A nivel local, la heterogeneidad espacial es otro factor importante que define su distribución y diversidad (Zanini et al., 2009; Branco et al., 2014; Rodríguez-Flores y Carmona, 2018). A nivel específico, las cianobacterias están presentes en condiciones ecológicas particulares; por ejemplo, Nostoc verrucosum Vaucher ex Gomont \& Flahault en aguas calcáreas, oligotróficas (Aboal, 1988; Mollenhouer et al., 1999) y Phormidium autumnale Gomont se ha registrado en ríos con temperatura mayor a 12 ${ }^{\circ} \mathrm{C}$, concentraciones altas de nitrato y ríos con descargas de agua mayores (Rodríguez-Flores y Carmona, 2018).

El objetivo de este estudio es contribuir al conocimiento ficoflorístico de cianobacterias bentónicas de cin- co ríos de la región central de México. Se presentan las descripciones morfológicas y ecológicas de las especies, se relaciona su distribución con los factores ambientales y microambientales, se analiza la relación de sus formas de crecimiento con factores microambientales (radiación fotosintéticamente activa, velocidad de corriente y profundidad) y se incluye la estimación de su diversidad. Por último, se discute la validez de la caracterización morfológica y ecológica en la delimitación de las especies de cianobacterias bentónicas de los ríos estudiados.

\section{Materiales y Métodos}

\section{Área de estudio}

El presente estudio se realizó en las siguientes regiones hidrológicas administrativas (RHA) de México:

1) RHA IV: Balsas, se ubica en el Estado de México, tiene un área de 112,039 km² y una extensión de $770 \mathrm{~km}$, es de orden siete con un escurrimiento natural de 16,363.2 $\mathrm{hm}^{3}$ por año y una precipitación normal anual promedio de 944 mm de 1981 a 2010 (CONAGUA, 2018). La parte central de la región presenta un clima templado subhúmedo, con lluvias en verano y una temperatura media anual entre 5 y $18^{\circ} \mathrm{C}$ (Miranda, 2015).

2) RHA IX: Golfo Norte, incluye al río Pánuco en el sur del estado de San Luis Potosí, tiene un área de 88,814 $\mathrm{km}^{2}$ y una extensión de $510 \mathrm{~km}$, es de orden siete con un escurrimiento natural de $20,223.6 \mathrm{hm}^{3}$ por año y una precipitación normal anual de $855 \mathrm{~mm}$ de 1981 a 2010. El clima predominante en el sur de San Luis Potosí es cálido subhúmedo con lluvias en verano con una temperatura media anual entre 22 y $26^{\circ} \mathrm{C}$ (CICESE, 2020).

La RHA IV, Balsas, cuenta con 15 cuencas en el Estado de México; se recolectaron los ríos silíceos Borbollón y Carrizal en el municipio Valle de Bravo. La RHA IX, Golfo Norte, se compone de 77 cuencas en San Luis Potosí; se muestrearon los ríos calcáreos La Garita, Micos y Tambaque en los municipios Aquismón y Ciudad Valles (CONAGUA, 2010) (Fig. 1, Cuadro 1).

\section{Muestreo}

La elección de trabajar con las cianobacterias en los ríos Borbollón, Carrizal, Micos, Tambaque y La Garita se basó en los registros de su presencia y abundancia documen- 


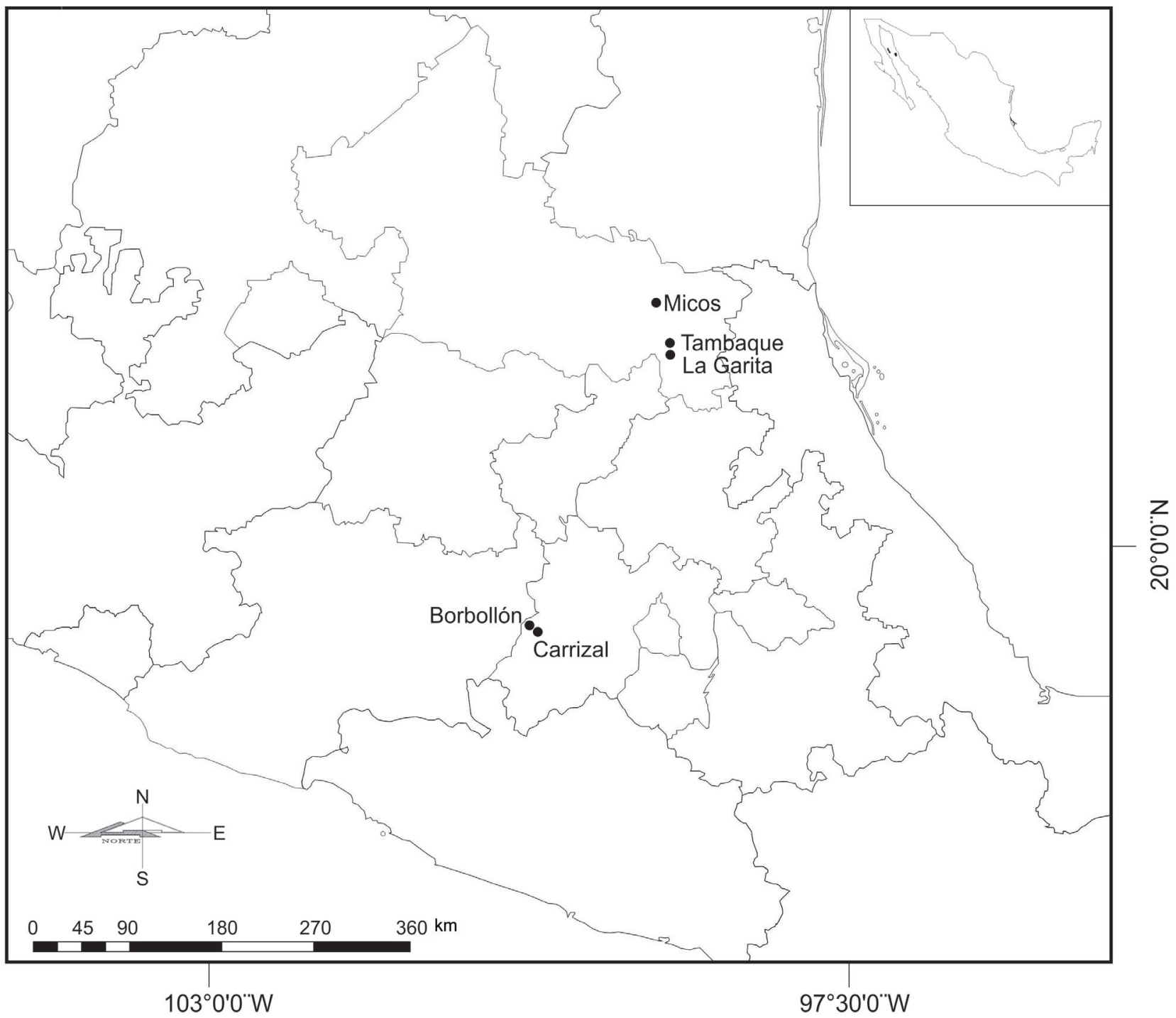

Figura 1: Ubicación de los ríos muestreados, con especies de cianobacterias bentónicas: ríos silíceos Borbollón y Carrizal, Estado de México, México, Región Hidrológica Administrativa IV, y ríos calcáreos La Garita, Micos y Tambaque, San Luis Potosí, Región Hidrológica Administrativa IX.

Cuadro 1: Ubicación, tipo y orden de los ríos silíceos Borbollón y Carrizal, municipio Valle de Bravo, Estado de México, Región Hidrológica Administrativa IV (RHA IV) Balsas, México, y los ríos calcáreos La Garita, Micos y Tambaque, municipios Aquismón y Ciudad Valles, San Luis Potosí, Región Hidrológica Administrativa IX (RHA IV) Golfo Norte, México.

\begin{tabular}{|c|c|c|c|c|c|c|c|}
\hline Estado & Región & Río & Latitud & Longitud & Orden río & Altitud (m s.n.m.) & Tipo de río \\
\hline Estado de & RHA IV & Borbollón & $19^{\circ} 06^{\prime} 84.7^{\prime \prime} \mathrm{N}$ & $100^{\circ} 09^{\prime} 38.5^{\prime \prime O}$ & Cuarto & 2200 & Silíceo \\
\hline México & RHA IV & Carrizal & $19^{\circ} 09^{\prime} 39.4^{\prime \prime} \mathrm{N}$ & $100^{\circ} 09^{\prime} 0.17^{\prime \prime O}$ & Cuarto & 2000 & Silíceo \\
\hline \multirow[t]{3}{*}{ San Luis Potosí } & RHA IX & Micos & $22^{\circ} 06^{\prime} 0.3^{\prime \prime} \mathrm{N}$ & $99^{\circ} 09^{\prime} 8.1^{\prime \prime} \mathrm{O}$ & Quinto & 120 & Calcáreo \\
\hline & RHA IX & Tambaque & $21^{\circ} 45^{\prime} 14.5^{\prime \prime} \mathrm{N}$ & $99^{\circ} 02^{\prime} 30.5^{\prime \prime O}$ & Cuarto & 150 & Calcáreo \\
\hline & RHA IX & La Garita & $21^{\circ} 40^{\prime} 33.3^{\prime \prime} \mathrm{N}$ & $99^{\circ} 02^{\prime} 40.5^{\prime \prime O}$ & Segundo & 60 & Calcáreo \\
\hline
\end{tabular}


tados en Cartajena (2004) y Bojorge-García et al. (2010). Además, se seleccionaron por: 1) ser ríos permanentes, 2) estar ubicados en dos regiones geográficas y 3) presentar distintas formas de crecimientos de cianobacterias bentónicas.

Las recolectas se llevaron a cabo en fechas diferentes en las dos RHA. Los ríos de la RHA IV, en el municipio Valle de Bravo, se muestrearon en siete ocasiones durante los años 2008 y 2009 en inicio de lluvias (LI, julio o septiembre), secas frías (SF, febrero, noviembre o diciembre) y secas cálidas (SC, mayo); mientras que los de la RHA IX, en los municipios Aquismón y Ciudad Valles, fueron recolectados cuatro veces durante los años 2009, 2010 y 2011, en secas ( $S$, noviembre, diciembre o febrero). En estos ríos, la temperatura del agua fue similar en todas las recolectas, por ello se reportaron como secas (Cuadro 2).

Para efectos de colecta, le llamamos río al transecto de $10 \mathrm{~m}$ de longitud en donde se hicieron los muestreos. Las formas de crecimiento de las especies fueron reconocidas como películas, fascículos, costras y colonias mucilaginosas (Sheath y Cole, 1992; Komárek y Anagnostidis, 2005). Los ríos fueron divididos cada dos metros; previo a la toma de muestras se estimó visualmente la cobertura de las formas de crecimiento con una unidad circular de muestreo con radio de $10 \mathrm{~m}$ (área $157 \mathrm{~m}^{2}$ ) (Necchi et al., 1995; BojorgeGarcía et al., 2010).

En el presente estudio seguimos el concepto de asociación como un conjunto de especies que forman una estructura física contínua, muestreadas juntas de forma regular y que coexisten en las mismas condiciones ambientales, espaciales y temporales (Margalef, 1983; Montoya y Aguirre, 2013).

\section{Análisis físico-químicos}

Los parámetros físico-químicos registrados in situ fueron temperatura del agua, conductividad específica $\left(\mathrm{K}_{25}\right)$ y $\mathrm{pH}$, medidos con un potenciómetro (Conductronic PC-18 Puebla, México). Se tomaron dos muestras de agua para la determinación de iones mayores, la primera de 1 I preservada a $4{ }^{\circ} \mathrm{C}$ en oscuridad, y la segunda de $0.5 \mathrm{I}$ acidificada con ácido nítrico a $\mathrm{pH}$ 2-3. El análisis de iones mayores $\left(\mathrm{Ca}^{2+}, \mathrm{Mg}^{2+}\right.$, $\mathrm{Na}^{2+}, \mathrm{K}^{+}, \mathrm{Si}_{-} \mathrm{SiO}_{2}, \mathrm{HCO}_{3}, \mathrm{Cl}^{-}$y $\mathrm{SO}_{4}{ }^{3-}$ ) se llevó a cabo en el Laboratorio de Química Analítica del Instituto de Geofísica de la Universidad Nacional Autónoma de México (UNAM). Con base en la mayor concentración de los compuestos disueltos en el agua, los ríos fueron designados como silíceos cuando el compuesto de mayor concentración fue el $\mathrm{Si}_{-} \mathrm{SiO}_{2}$ y calcáreos cuando el abundante fue el $\mathrm{Ca}^{2+}$ (Allan y Castillo, 2007).

Cuadro 2: Fechas de muestreo de los ríos silíceos Borbollón y Carrizal, municipio Valle de Bravo, Estado de México, Región Hidrológica Administrativa IV (RHA IV) Balsas, México, y los ríos calcáreos La Garita, Micos y Tambaque, municipios Aquismón y Ciudad Valles, San Luis Potosí, Región Hidrológica Administrativa IX (RHA IV) Golfo Norte, México. Secas frías=SF, Secas cálidas=SC, S=Secas, Ll=inicio de lluvias, 2008=08, 2009=09, 2010=10, $2011=11$.

\begin{tabular}{|c|c|c|c|c|}
\hline Estado & Región & Río & Secas cálidas (SC)/Secas frías (SF) & Lluvias (니) \\
\hline \multirow[t]{4}{*}{ Estado de México } & Balsas & Borbollón & Febrero 2009 (SF109) & Julio 2008 (LI108) \\
\hline & (RHA IV) & Carrizal & Mayo 2009 (SC09) & Septiembre 2008 (LI208) \\
\hline & & & Noviembre 2008 (SF108) & Septiembre 2009 (LI109) \\
\hline & & & Diciembre 2009 (SF209) & \\
\hline \multirow[t]{4}{*}{ San Luis Potosí } & Golfo Norte & Micos & Febrero 2010 (S10) & $\begin{array}{c}\text { No se muestreó en } \\
\text { Iluvias }\end{array}$ \\
\hline & (RHA IX) & Tambaque & Noviembre 2009 (S09) & $\begin{array}{c}\text { No se muestreó en } \\
\text { Iluvias }\end{array}$ \\
\hline & & La Garita & Noviembre 2010 (S10) & $\begin{array}{c}\text { No se muestreó en } \\
\text { Iluvias }\end{array}$ \\
\hline & & & Noviembre 2011 (S11) & $\begin{array}{c}\text { No se muestreó en } \\
\text { Iluvias }\end{array}$ \\
\hline
\end{tabular}


La toma de muestras para el análisis de nutrientes $\left(\mathrm{NH}_{4}{ }^{+}, \mathrm{NO}_{2}, \mathrm{NO}_{3}{ }^{2-}, \mathrm{PO}_{4}{ }^{3-}\right)$ se realizó a partir del filtrado in situ de $0.5 \mathrm{I}$ de agua con filtros de nitrocelulosa de $0.45 \mu \mathrm{m}$ y $0.22 \mu \mathrm{m}$ (Millipore, Massachusetts, EUA), posteriormente se guardaron en botellas estériles de polipropileno y se almacenaron a $4{ }^{\circ} \mathrm{C}$. La determinación se realizó con un analizador de Flujo Segmentado (Skalar San Plus System, Breda, Holanda) en el Laboratorio de Biogeoquímica Acuática del Instituto de Ciencias del Mar y Limnología, UNAM. La concentración de nitrógeno inorgánico fue registrada como la suma de concentraciones de amonio, nitrito y nitrato; es decir, nitrógeno inorgánico disuelto (NID), mientras que el fósforo como fósforo reactivo soluble (PRS). La designación del grado de trofía de los ríos se realizó con base en lo descrito por Dodds et al. (1998).

\section{Caracterización del hábitat y microhábitat}

Se realizó la descripción de los ríos empleando los siguientes criterios: fisiografía del terreno, tamaño y disposición del sustrato, radiación solar y velocidad de corriente (Carmona y Montejano, 1993). Las características de los microhábitats fueron registradas in situ, en el centro de cada unidad de muestreo. La velocidad de corriente y la profundidad fueron medidas con el uso de un medidor de corriente (Swoffer 3100, Washington, EUA). La radiación fotosintéticamente activa PAR (por sus siglas en inglés) fue medida con un sensor subacuático plano (Li-Cor LI-1000, Nebraska, EUA). En las diferentes fechas de recolecta el registro de la luz se realizó a la misma hora para cada río. El porcentaje de sustrato donde se establecieron las formas de crecimiento fue estimado de forma visual y se registraron como rocas, cantos rodados, grava, arena y limo, de acuerdo con la escala granulométrica de Wentworth (Allan y Castillo, 2007).

\section{Diversidad de cianobacterias}

La diversidad fue estimada a partir del índice de diversidad Shannon-Weaver $H=\Sigma\left[\left(p_{i}\right) \times\left(\ln p_{i}\right)\right]$ donde $p_{i}=$ abundancia en porcentaje de cada especie. La abundancia relativa de las especies fue medida en porcentaje y determinada a partir de $1 \mathrm{~cm}^{2}$; se designó como especie estructural a aquella más abundante en la asociación y como asociada a aquella con menor porcentaje. De acuerdo con Meave del Castillo et al. (2012), las especies fueron caracterizadas como raras $\leq 10 \%$, escasas $10-30 \%$, comunes $30-80 \%$ y abundantes $\geq 80 \%$. Para los análisis taxonómicos se empleó un microscopio estereoscópico (Olympus SZX7, Tokio, Japón) y un microscopio óptico (Olympus BX51, Tokio, Japón), ambos equipados con una cámara SC35 con sistema de microfotografía.

Para la actualización nomenclatural se empleó AlgaeBase (Guiry y Guiry, 2020) y en el arreglo filogenético de órdenes y familias, a Komárek et al. (2014). Además, se compararon caracteres taxonómicos para géneros y especies de los órdenes Nostocales, Oscillatoriales y Synechococcales (Geitler, 1932; Mollenhauer et al., 1999; John et al., 2003; Komárek y Anagnostidis, 2005; Jubera et al., 2009; Hrouzek et al., 2013; Komárek, 2013).

\section{Análisis estadísticos}

Puesto que los datos no mostraron distribución normal ni varianzas homogéneas, se utilizó la prueba de Kruskal-Wallis para evaluar si había diferencias significativas entre los datos microambientales. Las diferencias pareadas entre los datos fueron determinados con el test de Mann-Whitney $(p \leq 0.05)$. Las relaciones entre el porcentaje de cobertura de las especies y los parámetros ambientales fueron evaluadas mediante un análisis de redundancia (RDA). Los análisis fueron realizados con el programa XLSTAT (Addinsoft, 2013).

\section{Resultados}

\section{Composición taxonómica}

En total hubo nueve especies de cianobacterias en los ríos estudiados (Fig. 2). La diversidad fue baja, el número promedio de especies por sitio fue de $1.8( \pm 0.44)$ (Cuadro $3)$. El mayor número de especies (cuatro) correspondió al orden Oscillatoriales, seguido de tres de Nostocales y dos de Synechococcales. En cada río estuvieron presentes especies diferentes. Se reconocieron dos grupos de especies con base en las características ambientales. El primer grupo incluyó Phormidium lividum-Leptolyngbya truncata (PI-Lt) y Nostoc parmelioides-Coleodesmium wrangelii (Np-Cw) en los ríos silíceos de montaña, mientras que el segundo grupo estuvo compuesto por Schizothrix mexicana-Phormidium subfuscum (Sm-Ps), Homoeothrix juliana-Heteroleibleinia fontana (Hj-Hf) y Nostoc verrucosum (Nv) en los ríos calcáreos cálidos. 

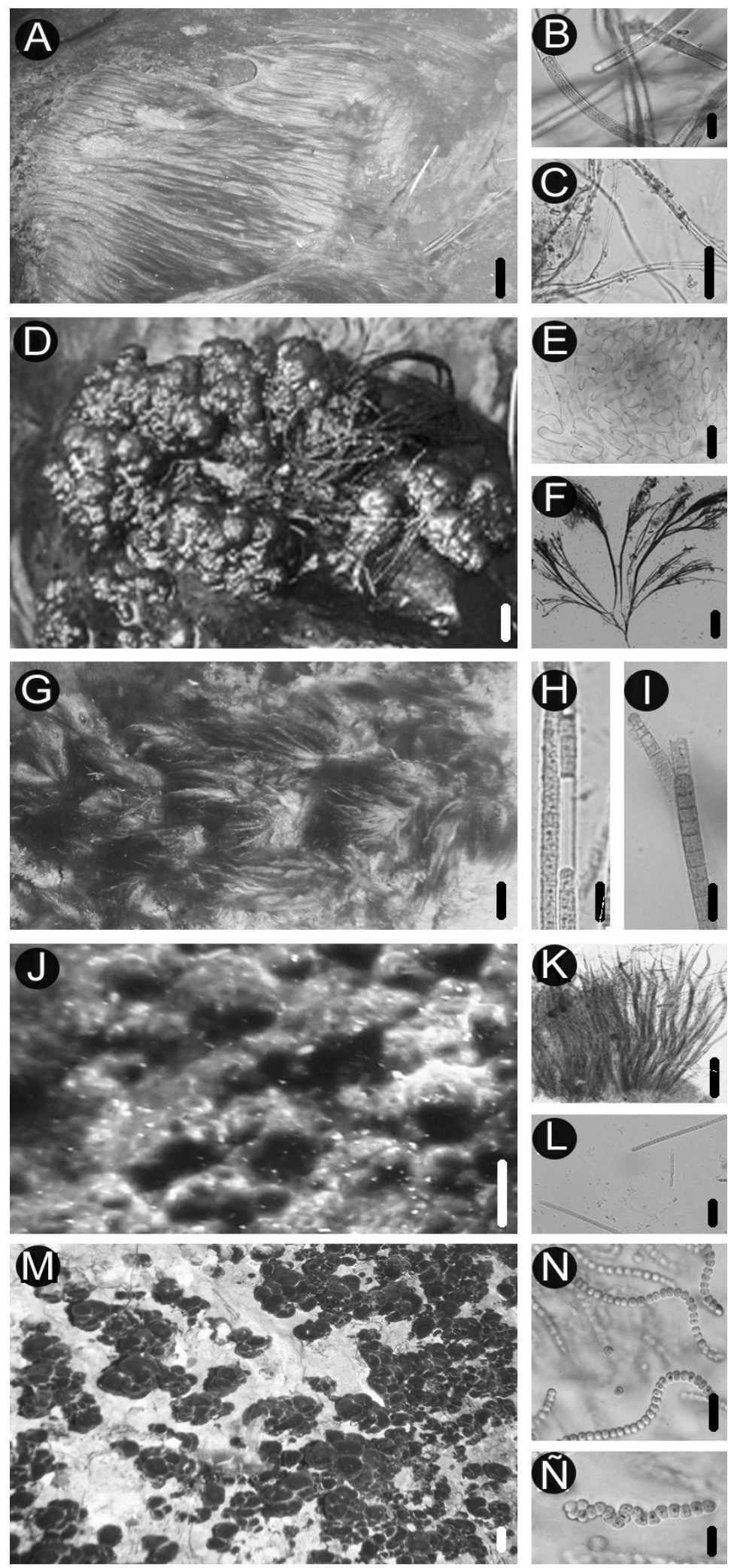

Figura 2: Formas de crecimiento y taxa de cianobacterias encontradas en cinco ríos, dos silíceos en el Estado de México, México, y tres calcáreos en el de San Luis Potosí, México. A. películas: B. Phormidium lividum (Hansgirg) Forti; C. Leptolyngbya truncata (Lemmermann) Anagnostidis \& Komárek. D. colonias mucilaginosas laxas: E. Nostoc parmelioides Kützing ex Bornet \& Flahault; F. Coleodesmium wrangelii Borzì ex Geitler. G. fascículos: H. Schizothrix mexicana Gomont; I. Phormidium subfuscum Kützing ex Gomont. J. costras: K. Homoeothrix juliana (Bornet \& Flahault ex Gomont) Kirchner; L. Heteroleibleinia fontana (Hansgirg) Anagnostidis \& Komárek. M. colonias mucilaginosas compactas: N. Nostoc verrucosum Vaucher ex Bornet \& Flahault. Ñ. etapas pluricelulares aseriadas. Escalas: $1 \mathrm{~cm}$ para las figuras A, D, G, J y M; $100 \mu \mathrm{m}$ para E, F, K; $10 \mu \mathrm{m}$ para B, C, H, I, L, N y Ñ. 


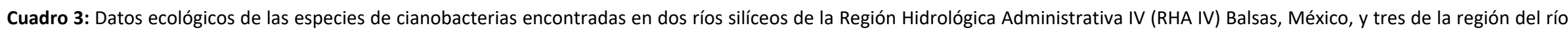

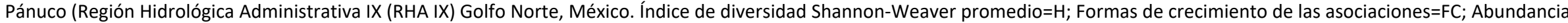
relativa promedio=ARP. Intervalo del mínimo y máximo de las variables microambientales: VC=Velocidad de corriente; Radiación fotosintéticamente activa=PAR; profundidad=Prof.

\begin{tabular}{|c|c|c|c|c|c|c|c|}
\hline Especies & H & FC & $\begin{array}{l}\text { ARP } \\
(\%)\end{array}$ & $\begin{array}{c}\text { VC } \\
\left(\mathrm{m} \mathrm{sg}^{-1}\right)\end{array}$ & $\begin{array}{c}\text { PAR } \\
\left(\mu \mathrm{mol} \text { fotones } \mathrm{m}^{2} \mathrm{sg}^{-1}\right)\end{array}$ & $\begin{array}{l}\text { Prof. } \\
\text { (m) }\end{array}$ & $\begin{array}{l}\text { Etapas de } \\
\text { desarrollo }\end{array}$ \\
\hline $\begin{array}{l}\text { Phormidium lividum (Hansgirg) } \\
\text { Forti } \\
\text { Leptolyngbya truncata } \\
\text { (Lemmermann) Anagnostidis } \\
\text { \& Komárek }\end{array}$ & 0.41 & Películas & $\begin{array}{l}48 \\
52\end{array}$ & $0.04-0.7$ & $13-826$ & $0.1-0.3$ & $\begin{array}{l}\text { Filamentos } \\
\text { jóvenes y viejos, } \\
\text { hormogonios } \\
\text { Filamentos jóvenes }\end{array}$ \\
\hline $\begin{array}{l}\text { Coleodesmium wrangelii Borzì } \\
\text { ex Geitler }\end{array}$ & 0.02 & $\begin{array}{l}\text { Colonias } \\
\text { mucilaginosas } \\
\text { laxas }\end{array}$ & $\begin{array}{l}98 \\
2\end{array}$ & $0.01-0.8$ & 238-1304 & $0.01-0.28$ & $\begin{array}{c}\text { Tricomas, } \\
\text { hormogonios, } \\
\text { acinetos y } \\
\text { heterocitos libres } \\
\text { Filamentos jóvenes } \\
\text { con heterocitos }\end{array}$ \\
\hline $\begin{array}{l}\text { Schizothrix mexicana Gomont } \\
\text { Phormidium subfuscum Kützing } \\
\text { ex Gomont }\end{array}$ & 0.34 & Fascículos & $\begin{array}{l}82 \\
18\end{array}$ & $0.17-0.36$ & $96-187$ & $0.09-0.17$ & $\begin{array}{c}\begin{array}{c}\text { Filamentos jóvenes, } \\
\text { hormogonios }\end{array} \\
\text { Filamentos jóvenes }\end{array}$ \\
\hline $\begin{array}{l}\text { Homoeothrix juliana (Bornet \& } \\
\text { Flahault ex Gomont) Kirchner } \\
\text { Heteroleibleinia fontana } \\
\text { (Hansgirg) Anagnostidis } \\
\text { \& Komárek }\end{array}$ & 0.65 & Costras & $\begin{array}{l}52 \\
48\end{array}$ & $0.2-0.6$ & $800-1813$ & $0.3-0.35$ & $\begin{array}{l}\text { Filamentos } \\
\text { jóvenes con y } \\
\text { sin atenuación, } \\
\text { filamentos con } \\
\text { hormogonios y } \\
\text { hormogonios } \\
\text { atenuados } \\
\text { Filamentos jóvenes }\end{array}$ \\
\hline $\begin{array}{c}\text { Nostoc verrucosum Vaucher } \\
\text { ex Bornet \& Flahault }\end{array}$ & 0 & $\begin{array}{l}\text { Colonias } \\
\text { mucilaginosas } \\
\text { compactas }\end{array}$ & 100 & $0.8-1$ & $38-201$ & $0.09-0.19$ & $\begin{array}{l}\text { Tricomas, etapas } \\
\text { pluricelulares } \\
\text { aseriadas y } \\
\text { paquetes de } \\
\text { células tipo } \\
\text { seudoparénquima }\end{array}$ \\
\hline
\end{tabular}




\section{Descripciones morfológicas}

\section{Cyanobacteria}

\section{Cyanophyceae}

\section{Nostocales}

Nostocaceae

Nostoc parmelioides Kützing ex Bornet \& Flahault, Ann. Sc. Nat., Bot. Sep. Ser. 7: 219-220. 1888. TIPO: ALEMANIA. Hirschbach, Thüringer Wald, norte de Schleusingen, ríos de montaña, 1830, F.T. Kützing (no localizado). Fig. 3.

Colonias mucilaginosas laxas, globulares o aplanadas, pardo-amarillentas, $0.8-5 \mathrm{~cm}$ de diámetro (Figs. 3A, 3B); mucílago común gelatinoso, laxo a firme; tricomas flexuosos (Fig. 3C), constreñidos (Figs. 3D, 3H), 2.5-7.6 $\mu \mathrm{m}$ de ancho (Figs. 3C, 3D), vaina presente o ausente; células vegetativas 2.1-7.7 $\mu \mathrm{m}$ de ancho, 2.5-6.8 $\mu \mathrm{m}$ de longitud (Fig. 3E), isodiamétricas o ligeramente más largas que anchas; heterocitos esféricos, libres, basales y/o intercalares 3.2-11.8 $\mu \mathrm{m}$ de ancho, 3.7-15 $\mu \mathrm{m}$ de longitud (Fig. 3E); no se observaron etapas pluricelulares aseriadas; acinetos elípticos 4-7 $\mu \mathrm{m}$ de ancho, 5.2-9 $\mu \mathrm{m}$ de longitud (Fig. 3F), 1.2-2.1 más largos que anchos, germinación de los acinetos con 1-4 células, 11.5-13.5 $\mu \mathrm{m}$ de ancho (Fig. 3G); paquetes de células tipo seudoparénquima ausentes.

Etapas de desarrollo: el fin de lluvias fue la época de mayor crecimiento y las colonias estuvieron compuestas por hormogonios y heterocitos libres que produjeron mucílago suave y laxo. En el inicio de lluvias los acinetos libres y sus etapas de germinación fueron abundantes (Figs. 3F, $3 G)$.

Hábitat: temperatura del agua $10-17{ }^{\circ} \mathrm{C} ; \mathrm{K}_{25} 52-127$ $\mu \mathrm{S} \mathrm{cm}{ }^{-1}$; pH 6.9-7.8; velocidad de corriente 0.01-0.8 $\mathrm{m} \mathrm{s}^{-1}$; PAR 238-1304 $\mu \mathrm{mol}$ fotón $\mathrm{m}^{-2} \mathrm{~s}^{-1}$; profundidad 0.1-0.28 m; sustrato roca madre emergente $59 \%$, roca sumergida $40 \%$, tronco $1 \%$. Epilítica, sobre la base de sus colonias crece en río Carrizal la especie asociada Coleodesmium wrangelii Borzì ex Geitler.

Ejemplares examinados: MÉXICO. Estado de México, municipio Valle de Bravo, Carrizal, 21.VII.2008, 08.IX.2008, 29.XI.2008, 6.II.2009, 8.IX.2009, 5.XII.2009, J. Carmona y M. Cartajena VB150 (FCME), VB167 (FCME).

Distribución: Argentina, Río Negro; Estados Unidos de América, Arizona, Carolina del Norte, Connecticut, Nueva Inglaterra, Pensilvania, Texas y Wyoming; México, Estado de México y Morelos; Noruega; Perú, la región central andina; Puerto Rico; Suecia.

Ecología: arroyos, estanques, ríos y suelos; formas de vida edáfica y epilítica.

Nostoc verrucosum Vaucher ex Bornet \& Flahault, Ann. Sc. Nat., Bot. Sep. Ser. 7: 216-219. 1888. TIPO: SUIZA. Geneva, departamento Meurthe, Moselle, Rambervillers, río calcáreo, sobre rocas, VIII.1823. J.B. Mougeot y Ch. G. Nestler 798 (no localizado). Fig. 4.

Colonias mucilaginosas compactas, globulares, yemas, negro-violáceas, consistencia gelatinosa firme, $0.8-3 \mathrm{~cm}$ de diámetro (Fig. 4A), mucílago común denso, lila; tricomas constreñidos (Figs. 4B, 4C), vaina presente o ausente; células vegetativas esféricas (Fig. 4D) 3-7.8 $\mu \mathrm{m}$ de ancho, 1.5-6.5 $\mu \mathrm{m}$ de longitud, isodiamétricas; heterocitos esféricos, libres, (Fig. 4D), basales y/o intercalares (Figs. 4C, 4E), 4.3-6.5 $\mu \mathrm{m}$ de ancho, 3.3-4.2 $\mu \mathrm{m}$ de longitud (Fig. 4E); etapas pluricelulares aseriadas con heterocito basal compuestas por 1-3 células (Figs. 4E, 4F); no se observaron acinetos; paquetes de células tipo seudoparénquima presentes (Figs. 4G, 4H).

Etapas de desarrollo: tricomas, etapas pluricelulares aseriadas y hormogonios estuvieron en porcentajes similares en todos los muestreos. Los paquetes de células tipo seudoparénquima sólo se colectaron una vez.

Hábitat: temperatura del agua $20-26{ }^{\circ} \mathrm{C} ; \mathrm{K}_{25} 515-$ $1430 \mu \mathrm{S} \mathrm{cm}^{-1}$; $\mathrm{pH} 7.4-8.2$; velocidad de corriente 0.8-1 $\mathrm{m} \mathrm{s}^{-1}$; PAR 38-201 $\mu \mathrm{mol}$ fotón $\mathrm{m}^{-2} \mathrm{~s}^{-1}$; profundidad 0.09-0.19 m; 


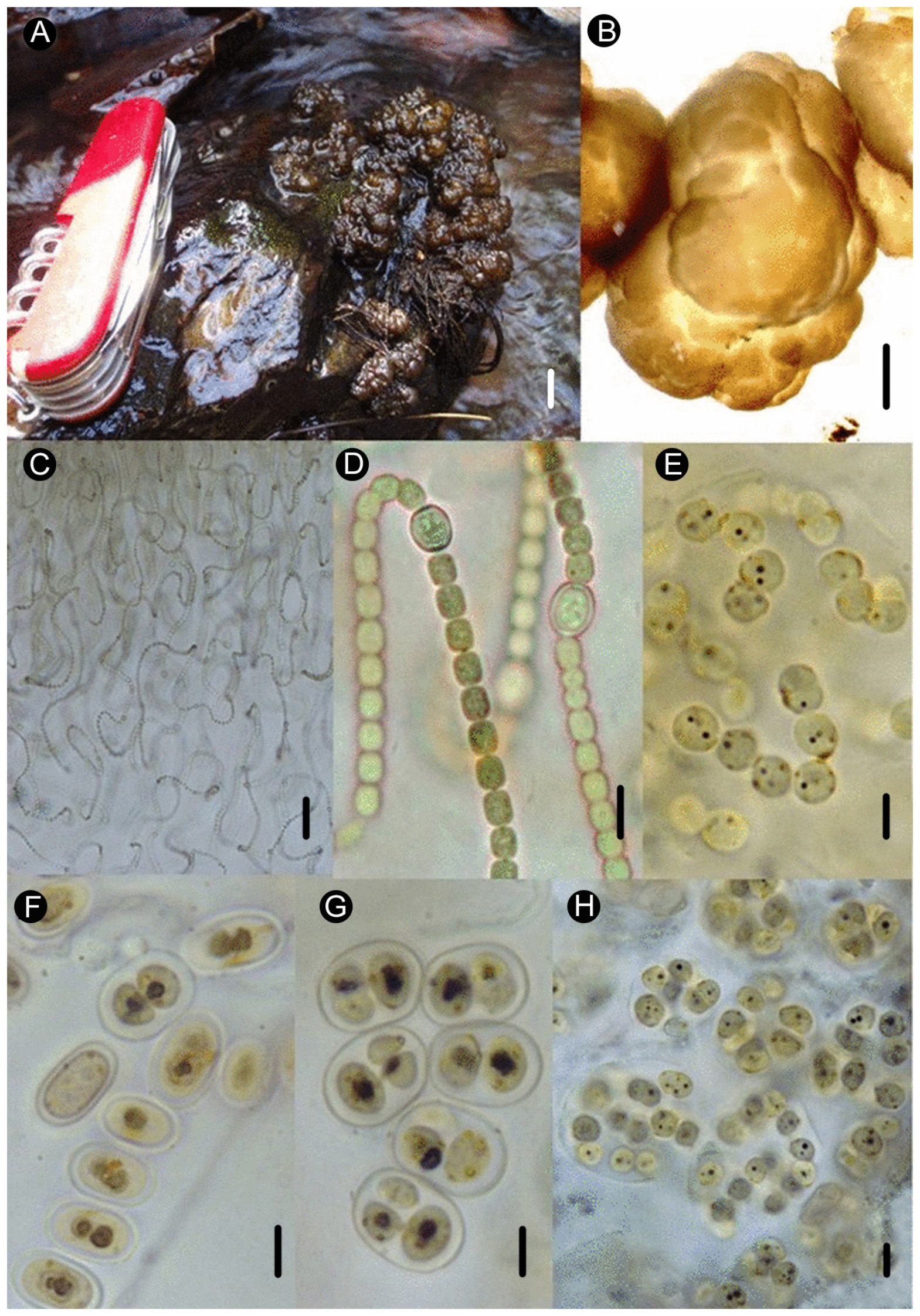

Figura 3: Nostoc parmelioides Kützing ex Bornet \& Flahault. A. y B. colonias mucilaginosas laxas; C. disposición de los tricomas; D. acercamiento de tricomas con heterocito intercalar; $\mathrm{E}$. células del tricoma con gránulos de polifosfato; F. acinetos dispuestos en hilera y con gránulos de polifosfato; $\mathrm{G}$. germinación de los acinetos; H. colonias microscópicas. Escalas: $5 \mathrm{~cm}$ para la figura A; $0.5 \mathrm{~cm}$ para B; $10 \mu \mathrm{m}$ para C y D; $5 \mu \mathrm{m}$ para E, F, G y H. 


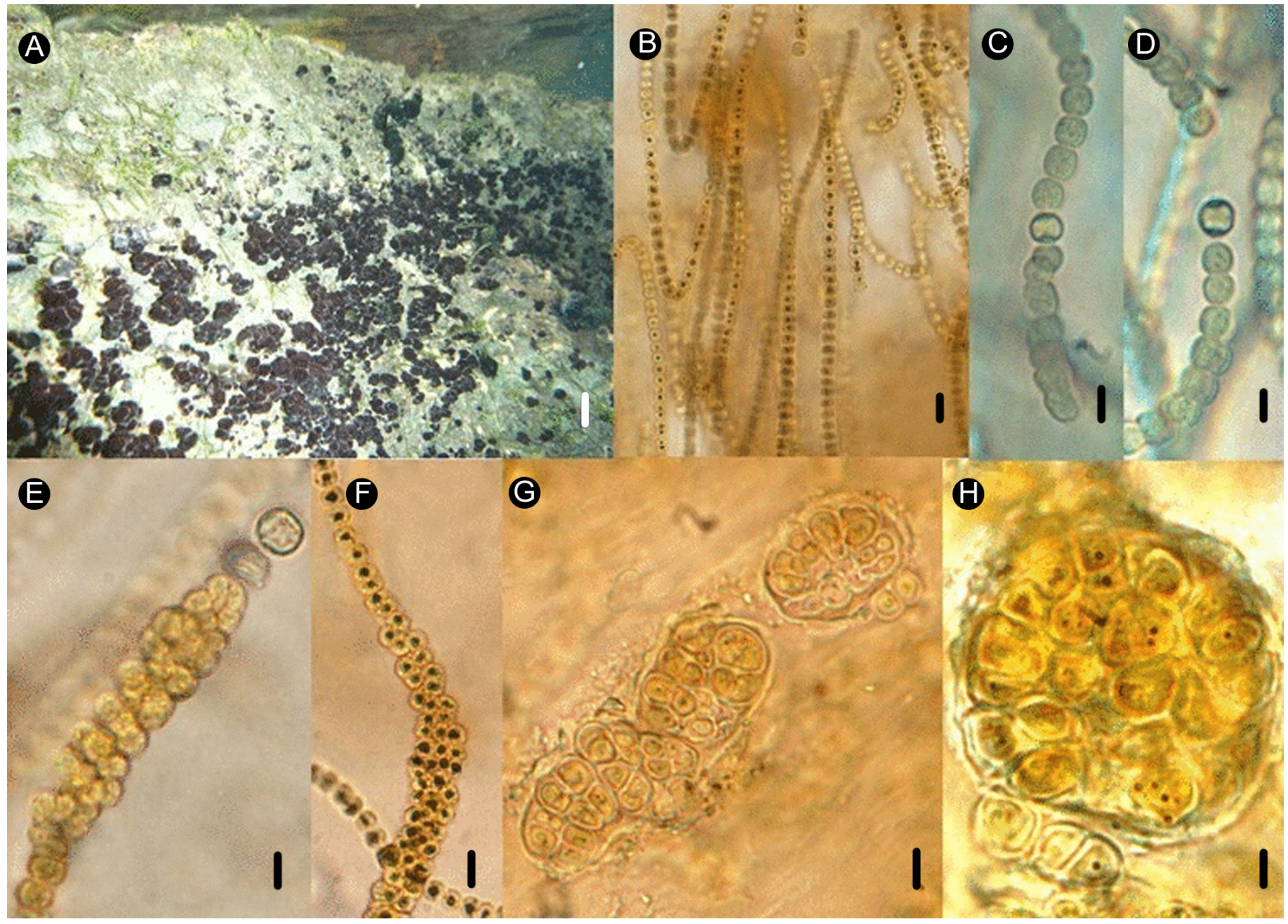

Figura 4: Nostoc verrucosum Vaucher ex Bornet \& Flahault. A. colonias mucilaginosas compactas gregarias sobre la roca; B. disposición de los tricomas; C. tricoma con heterocito intercalar; D. heterocito en la base del tricoma; E. fase pluriseriada con heterocito basal; F. etapa pluriseriada aseriada atenuada con gránulos de polifosfato; G.-H. fases esféricas tipo seudoparénquima. Escalas: $3 \mathrm{~cm}$ para la figura A; $15 \mu \mathrm{m}$ para B; $8 \mu \mathrm{m}$ para C, D y E; $10 \mu \mathrm{m}$ para G y H.

sustrato roca $100 \%$. Epilítica, no formó asociación con ninguna otra cianobacteria, crece entremezclada con musgos.

Ejemplares examinados: MÉXICO. San Luis Potosí, municipio Aquismón, La Garita, 9.II.2009, 24.XI.2009, 17.XI.2010, 21.XI.2011, J. Carmona y M. Cartajena PA4350 (FCME), PA4370 (FCME).

Distribución: Argentina, Buenos Aires, Córdoba, Neuquén; Colombia, Bogotá; Costa Rica; Estados Unidos de América, Arizona, Illinois, Montana, Pensilvania y Wyoming; México, Estado de México, Puebla, San Luis Potosí, Tamaulipas y Yucatán; España, Albacete, Alicante, Jaén y Murcia.
Ecología: arroyos, charcos, manantiales, ríos y suelo húmedo; formas de vida epilítica y edáfica.

\section{Tolypothrichaceae}

Coleodesmium wrangelii Borzì ex Geitler, Nat. Pfl. Sw. Auf. 1: 356. 1942. TIPO: ESTADOS UNIDOS DE AMÉRICA. Nordstrom. Fig. 5.

Fascículos (Fig. 5A), ramas falsas (Fig. 5B) 0.5-0.9 $\mathrm{cm}$ de longitud, 1-2 tricomas en ramas basales, 2-3 ramas centrales con 6 tricomas, ramas apicales con 1-2 tricomas, filamentos heteropolares, 7.8-11.5 $\mu \mathrm{m}$ de ancho; tricomas constreñidos, paralelos 7.8-13.8 $\mu \mathrm{m}$ de ancho; vaina di- 


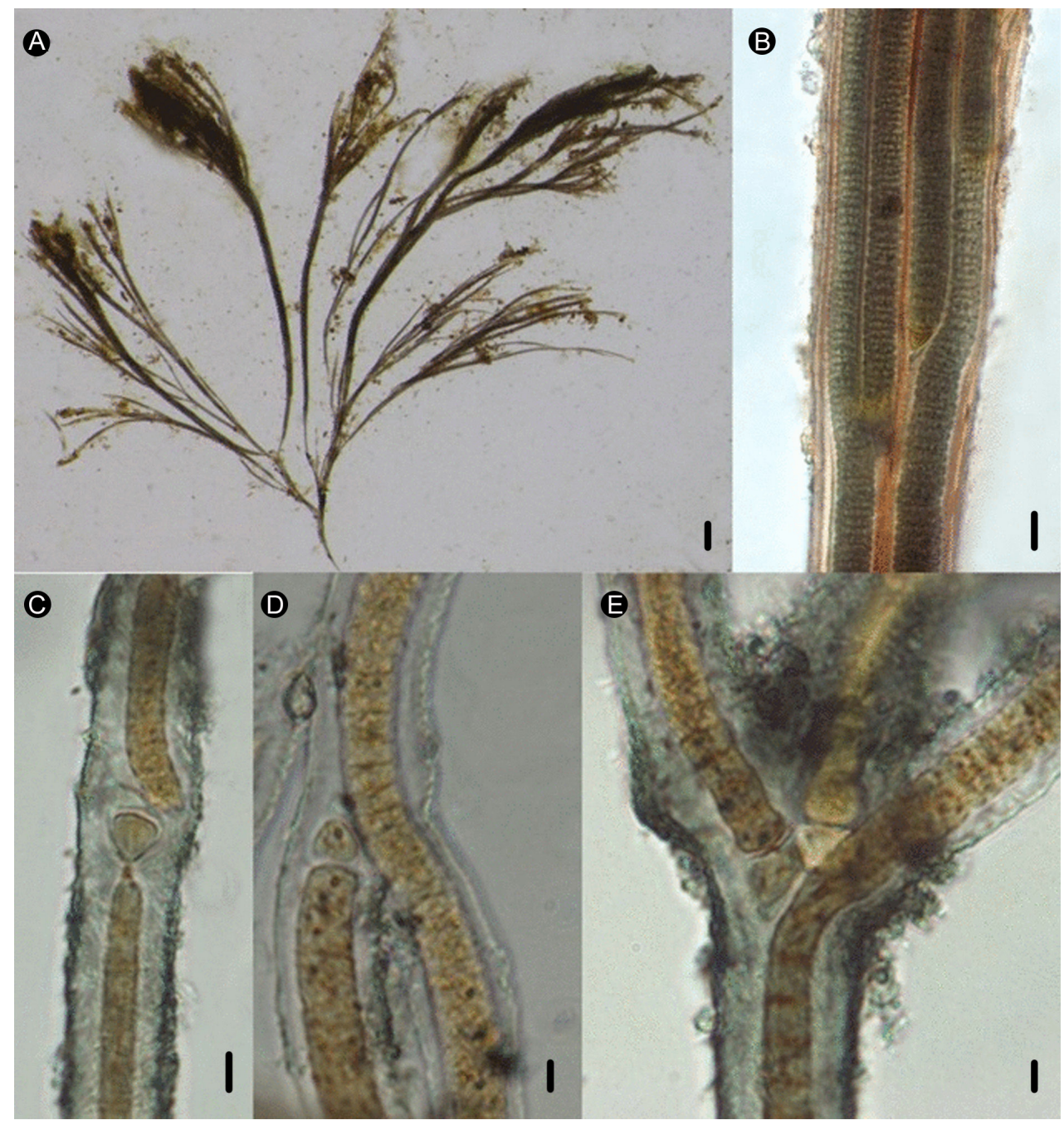

Figura 5: Coleodesmium wrangelii Borzì ex Geitler. A. fascículos compuestos por ramas falsas con varios tricomas por vaina; B. eje principal del fascículo con 4 tricomas; C. heterocito intercalar y vaina mucilaginosa; D. y E. acercamientos de la base de dos ramas con un heterocito. Escalas: 2 $\mathrm{mm}$ para la figura $\mathrm{A} ; 8 \mu \mathrm{m}$ para B y $\mathrm{C} ; 5 \mu \mathrm{m}$ para $\mathrm{D}$ y $\mathrm{E}$.

fluente e incolora; células vegetativas $8-11 \mu \mathrm{m}$ de ancho, 2.1-4 $\mu \mathrm{m}$ de longitud, 0.26-0.5 veces más cortas que anchas; heterocitos en la base de las ramas, subesféricos, 3.7-11.2 $\mu \mathrm{m}$ de ancho, 8-10.5 $\mu \mathrm{m}$ de longitud (Figs. 5D, 5E); célula apical redondeada, hemisférica; no se observaron acinetos.

Etapas de desarrollo: filamentos jóvenes con heterocitos en todas las recolectas (Figs. 5C, 5E).

Hábitat: temperatura del agua $10-17{ }^{\circ} \mathrm{C} ; \mathrm{K}_{25} 52-127$ $\mu \mathrm{S} \mathrm{cm}^{-1}$; pH 6.9-7.8 velocidad de corriente 0.01-0.8 $\mathrm{m} \mathrm{s}^{-1}$; PAR 238-1304 $\mu \mathrm{mol}$ fotón $\mathrm{m}^{-2} \mathrm{~s}^{-1}$; profundidad 0.01-0.28 m; sustrato roca madre emergente $59 \%$, roca sumergida $40 \%$, tronco 1\%. Epilítica, crece asociada a las colonias de Nostoc parmelioides.

Ejemplares examinados: MÉXICO. Estado de México, municipio Valle de Bravo, Carrizal, 21.VII.2008, 08.IX.2008, 29.XI.2008, 6.II.2009, 8.IX.2009, 5.XII.2009, J. Carmona y M. Cartajena VB122 (FCME), VB150 (FCME).

Distribución: Ártico; México, Cuenca de México y Huasteca Potosina; Europa Occidental; República Checa.

Ecología: arroyos, ríos de montaña, calcáreos y de corriente rápida y desiertos de la zona ártica; forma de vida epilítica. 
Oscillatoriales

\section{Oscillatoriaceae}

Phormidium lividum (Hansgirg) Forti, Syll. Alg. Omn. Huc. Cogn. 5: 230. 1907. TIPO: AUSTRIA. Carintia. Fig. 6.

Película delgada, suave, verde-azul intenso (Fig. 6A); filamentos rectos paralelos a irregulares, densamente entremezclados (Fig. 6B); vaina hialina, delgada, incolora (Fig. 6B) a mucilaginosa difluente (Fig. 6D), algunos individuos sin vaina, tricomas, verde claro a verde intenso, no atenuados, no contreñidos, 6.8-8.4 $\mu \mathrm{m}$ de ancho (Fig. 6B); células vegetativas 5.5-8.5 $\mu \mathrm{m}$ de ancho, 5-8 $\mu \mathrm{m}$ de longitud, 0.6-0.9 veces más largas que anchas, contenido celular homogéneo, sin hilera de gránulos alrededor de las paredes celulares; células apicales redondeadas, no atenuadas y sin caliptra (Fig. 6B), hormogonios libres.

Etapas de desarrollo: los filamentos con vaina hialina (Fig. 6B) y hormogonios (Fig. 6C) estuvieron en todas las épocas del año, los tricomas fueron abundantes en fin de Iluvias y secas frías y los filamentos con vaina difluente en inicio de lluvias.

Hábitat: temperatura del agua $10-18{ }^{\circ} \mathrm{C} ; \mathrm{K}_{25} 53-148$ $\mu \mathrm{S} \mathrm{cm}{ }^{-1}$; $\mathrm{pH}$ 6.9-7.6; velocidad de corriente 0.04-0.7 $\mathrm{m} \mathrm{s}^{-1}$;
PAR 13-826 $\mu \mathrm{mol}$ fotón $\mathrm{m}^{-2} \mathrm{~s}^{-1}$; profundidad 0.1-0.3 m; sustrato cantos rodados $91 \%$, grava $3 \%$ y rocas $6 \%$. Epilítica, compone el segundo estrato de la película que forma con Leptolyngbya truncata (Lemmermann) Anagnostidis \& Komárek.

Ejemplares examinados: MÉXICO. Estado de México, municipio Valle de Bravo, Borbollón, 20.VII.2008, 07.IX.2008, 28.XI.2008, 5.II.2009, 7.IX.2009, 4.XII.2009, J. Carmona y M. Cartajena VB120 (FCME), VB129 (FCME).

Distribución: Ártico, Francia, Inglaterra, Rusia y Ucrania.

Ecología: piscinas, rocas húmedas y suelos; formas de vida edáfica, epilítica y subaérea.

Phormidium subfuscum Kützing ex Gomont, Ann. Sc. Nat. Bot. Ser. 7, 16: 182, pl. V. 1892. TIPO: SUIZA. Sin localidad. Fig. 7.

Crece entre los filamentos de Schizothrix mexicana Gomont; filamentos rectos, paralelos, 4.3-8.5 $\mu \mathrm{m}$ de ancho (Fig. 7B); vaina hialina, firme, visible en la parte apical (Fig. 7B); tricomas violetas o verde-azules, atenuados en el ápice, no constreñidos (Fig. 7C); células vegetativas 6.4-9.1 $\mu \mathrm{m}$ de ancho, 4.7-11.1 $\mu \mathrm{m}$ de longitud, 0.6-1.4 veces más
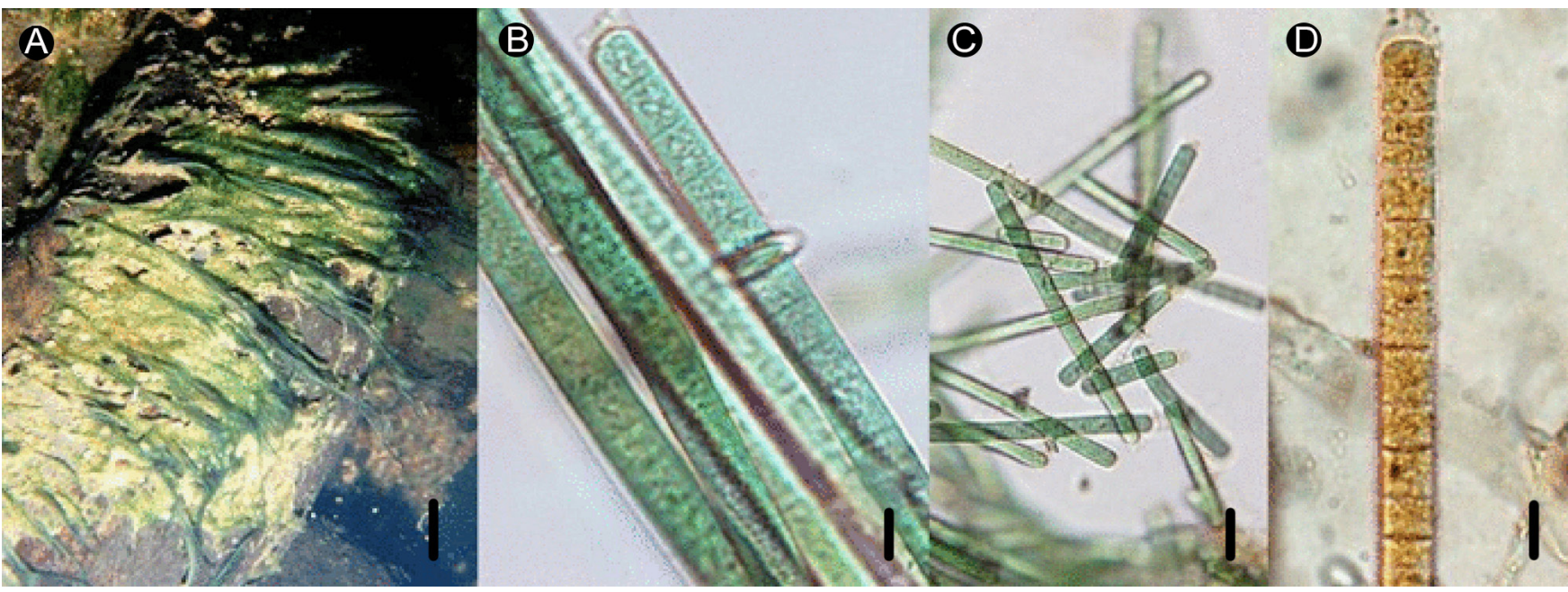

Figura 6: Phormidium lividum (Hansgirg) Forti. A. película en fin de lluvias; B. disposición alineada de los filamentos con vaina hialina; C. hormogonios; D. célula apical redondeada. Escalas: $10 \mathrm{~cm}$ para la figura A; $8 \mu \mathrm{m}$ para B y D; $15 \mu \mathrm{m}$ para C. 
largas que anchas, contenido celular homogéneo, con una hilera de gránulos alrededor de las paredes celulares (Figs. 7B, 7C), células apicales cónicas, atenuadas (Fig. 7D), 8-9.7 $\mu \mathrm{m}$ de ancho, 2.7-3.7 $\mu \mathrm{m}$ de longitud, caliptra cónica; no se observaron hormogonios.

Etapas de desarrollo: filamentos jóvenes en todos los muestreos (Figs. 7B-D).

Hábitat: temperatura del agua $20-26{ }^{\circ} \mathrm{C} ; \mathrm{K}_{25} 1209-$ $1476 \mu \mathrm{S} \mathrm{cm}^{-1}$; pH 7.7-7.9; velocidad de corriente 0.17-0.36 $\mathrm{m} \mathrm{s}^{-1}$; PAR 96-187 $\mu \mathrm{mol}$ fotón $\mathrm{m}^{-2} \mathrm{~s}^{-1}$; profundidad 0.09-0.17 m. Metafítica, crece entre los filamentos de Schizothrix mexicana Gomont.

Ejemplares examinados: MÉXICO. San Luis Potosí, municipio Ciudad Valles, Micos, 06.II.2009, 24.XI.2009, 18.XI.2011, J. Carmona y M. Cartajena PA4345 (FCME), PA4365 (FCME).

Distribución: Argentina, Córdoba; Brasil; Estados Unidos de América, Arizona, Carolina del Norte, Illinois;
México, San Luis Potosí, Sonora, Veracruz, Yucatán; Perú, Lima; Polonia.

Ecología: arroyos, aguas negras, cascadas, cenotes, estanques, lagos, manantiales, ríos, paredones, suelos; formas de vida edáfica, epilítica, epifita y planctónica.

\section{Homoeotrichaceae}

Homoeothrix juliana (Bornet \& Flahault ex Gomont) Kirchner, Nat. Pfl. Sw. Auf. 1: 348. 1898. TIPO: FRANCIA. Coupigny. Fig. 8.

Costras duras, quebradizas, con carbonato de calcio (Fig. 8A); filamentos heteropolares, paralelos (Fig. 8B), vainas firmes, hialinas (Fig. 8E), atenuación del filamento 20$37 \%$ de la longitud del filamento (Fig. 8D), tricomas verde olivo o violáceo, ligeramente constreñidos, 204-367 $\mu \mathrm{m}$ de longitud, células vegetativas 10.5-11.6 $\mu \mathrm{m}$ de ancho, 1-1.5 $\mu \mathrm{m}$ de longitud, 0.09-0.12 veces más largas que anchas, contenido celular granuloso (Fig. 8D), sin hilera de gránulos alrededor de las paredes; pelo hialino apical, angosto,

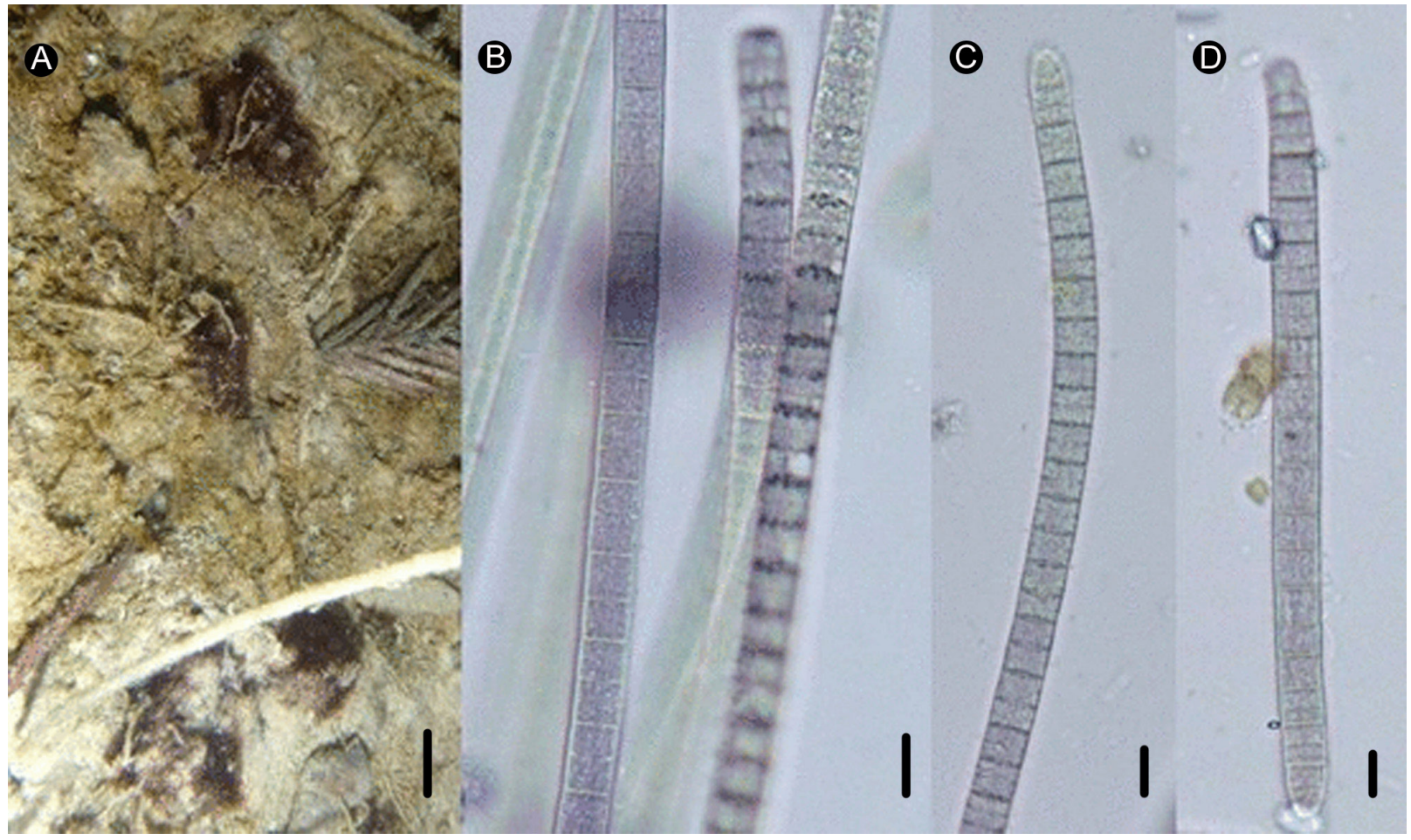

Figura 7: Phormidium subfuscum Kützing ex Gomont. A. fascículos donde $P$. subfuscum es metafitica; B. filamentos lilas y con gránulos alrededor de los septos celulares; C-D. célula apical atenuada. Escalas: $2 \mathrm{~cm}$ para la figura A; $8 \mu \mathrm{m}$ para B y $6 \mu \mathrm{m}$ para $C$ y D. 
2-6 $\mu \mathrm{m}$ de ancho, 25-55 $\mu \mathrm{m}$ de longitud, (Figs. 8C, 8D); células apicales vacuoladas, atenuadas, sin caliptra (Fig. 8D); hormogonios libres 8.5-10.1 $\mu \mathrm{m}$ de ancho, 35-108 $\mu \mathrm{m}$ de longitud.

Etapas de desarrollo: filamentos jóvenes con y sin atenuación (Fig. 8F), filamentos con hormogonios y hormogonios atenuados libres (Figs. 8C, 8E, 8F) se encontraron en porcentajes similares a inicio y fines de secas cálidas.

Hábitat: temperatura del agua $23-25^{\circ} \mathrm{C} ; \mathrm{K}_{25} 1120$ $1351 \mu \mathrm{S} \mathrm{cm}^{-1} ; \mathrm{pH}$ 7.8-8.2; velocidad de corriente 0.2-0.6 m $\mathrm{s}^{-1}$; PAR 800-1813 $\mu \mathrm{mol}$ fotón $\mathrm{m}^{-2} \mathrm{~s}^{-1}$; profundidad 0.3-0.35 $\mathrm{m}$; sustrato roca $100 \%$. Epilítica, forma asociaciones con los filamentos de Heteroleibleinia fontana (Hansgirg) Anagnostidis \& Komárek.

Ejemplares examinados: MÉXICO. San Luis Potosí, municipio Aquismón, Tambaque, 24.XI.2009, 17.XI.2010, 18.XI.2011, J. Carmona y M. Cartajena PA4349 (FCME), PA4369 (FCME).

Distribución: España, Albacete, Alicante, Jaen y Murcia; México, Michoacán, Morelos, San Luis Potosí y Tamaulipas.

Ecología: arroyos, lagos, manantiales calcáreos, manantiales de montaña, ríos; formas de vida bentónica, epilítica, epifita y perifítica.

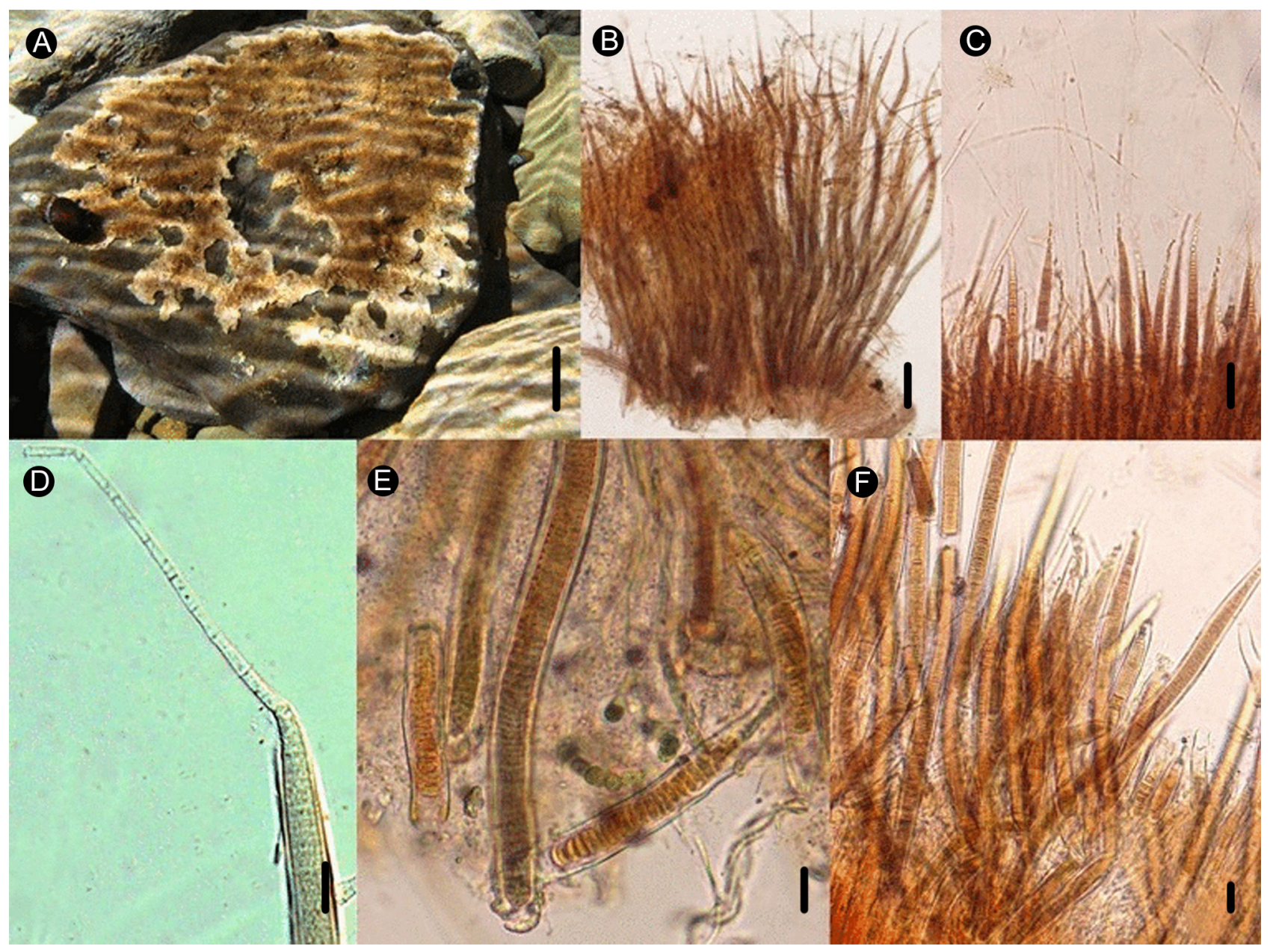

Figura 8: Homoeothrix juliana (Bornet \& Flahault ex Gomont) Kirchner. A. costra; B. filamentos heteropolares que componen la costra; C. atenuación de los filamentos tipo cabello y presencia de hormogonios atenuados en la parte apical de los filamentos; D. acercamiento de la atenuación de los filamentos; E. célula basal redondeada; F. filamentos entremezclados de Homoeothrix juliana (Bornet \& Flahault ex Gomont) Kirchner y Heteroleibleinia fontana (Hansgirg) Anagnostidis \& Komárek. Escalas: $10 \mathrm{~cm}$ para la figura A; $15 \mu \mathrm{m}$ para B y C; $10 \mu \mathrm{m}$ para D, E, y F. 


\section{Synechococcophycidae}

\section{Synechococcales}

Leptolyngbyaceae

Leptolyngbya truncata (Lemmermann) Anagnostidis \& Komárek, Arch. Hydr. Supp. 80: 393. 1988. TIPO: No conocido. Fig. 9.

Película delgada, compacta, suave (Figs. 9A, 9B); filamentos ligeramente ondulados, vaina hialina (Fig. 9C), tricomas verde-pálido, no atenuados, no constreñidos, 0.6-1.83 $\mu \mathrm{m}$ de ancho, células vegetativas más largas que anchas, contenido celular verde claro homogéneo (Figs. 9C, 9D), sin hilera de gránulos alrededor de las paredes celulares, células apicales truncadas, no atenuadas, sin caliptra, 0.4-0.5 $\mu \mathrm{m}$ de longitud, no se observaron hormogonios.

Etapas de desarrollo: filamentos jóvenes en todas las épocas del año (Figs. 9C, 9D).

Hábitat: temperatura del agua $10-18{ }^{\circ} \mathrm{C} ; \mathrm{K}_{25} 53-148$ $\mu \mathrm{S} \mathrm{cm}{ }^{-1}$; pH 6.9-7.6; velocidad de corriente 0.04-0.7 $\mathrm{m} \mathrm{s}^{-1}$; PAR 13-826 $\mu$ mol fotón $\mathrm{m}^{-2} \mathrm{~s}^{-1}$; profundidad 0.1-0.3 m; sustrato cantos rodados $91 \%$, grava $3 \%$ y rocas $6 \%$. Epilítica, conforma el primer estrato de las películas que forma con Phormidium lividum.
Ejemplares examinados: MÉXICO. Estado de México, municipio Valle de Bravo, Borbollón, 20.VII.2008, 07.IX.2008, 28.XI.2008, 5.II.2009, 7.IX.2009, 4.XII.2009, J. Carmona y M. Cartajena VB108 (FCME), VB116 (FCME).

\section{Distribución: Alemania, Grecia y Hungría.}

Ecología: arroyos y ríos; formas de vida epilítica, epífita y subaérea.

\section{Oculatellaceae}

Heteroleibleinia fontana (Hansgirg) Anagnostidis \& Komárek, Arch. Hydr. Supp. 80: 434. 1988. TIPO: REPÚBLICA CHECA. Elbeteinitz. Fig. 10.

Costras quebradizas con carbonato de calcio (Fig. 10A); filamentos heteropolares rectos, paralelos, delgados (Fig. 10B), vainas hialinas, firmes; tricomas verde-azul, no atenuados, ligeramente constreñidos (Fig. 10C), 0.6-1.8 $\mu \mathrm{m}$ de ancho, células vegetativas 1.2-2, 0.7-1 $\mu \mathrm{m}$ de ancho, 1.1-1.6 $\mu \mathrm{m}$ de longitud, más largas que anchas, contenido celular hialino homogéneo, sin hilera de gránulos alrededor de las paredes celulares; células apicales redondeadas, no atenuadas, sin caliptra, 0.5-0.6 $\mu \mathrm{m}$ de longitud; no se observaron hormogonios.

Etapas de desarrollo: filamentos jóvenes en todos los muestreos (Fig. 10C).

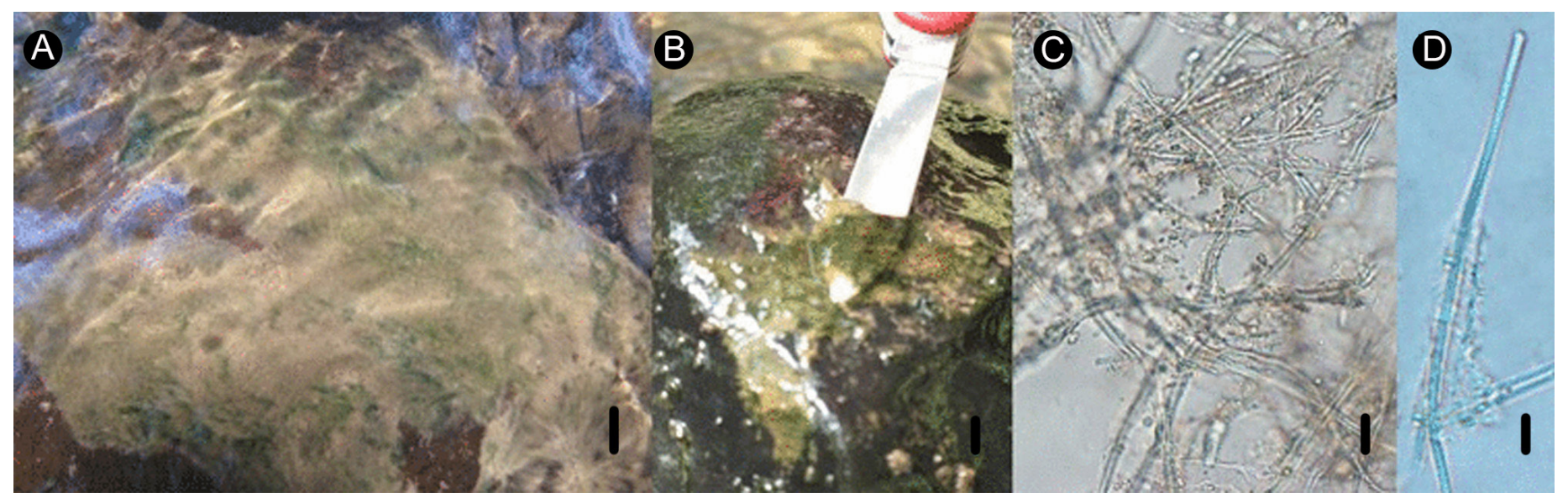

Figura 9: Leptolyngbya truncata (Lemmermann) Anagnostidis \& Komárek. A. película en época de secas; B. película en época de lluvias; C. disposición entrelazada de los filamentos; D. filamento con célula apical redondeada. Escalas: $5 \mathrm{~cm}$ para las figuras A y B; $3 \mu \mathrm{m}$ para C y $D$. 

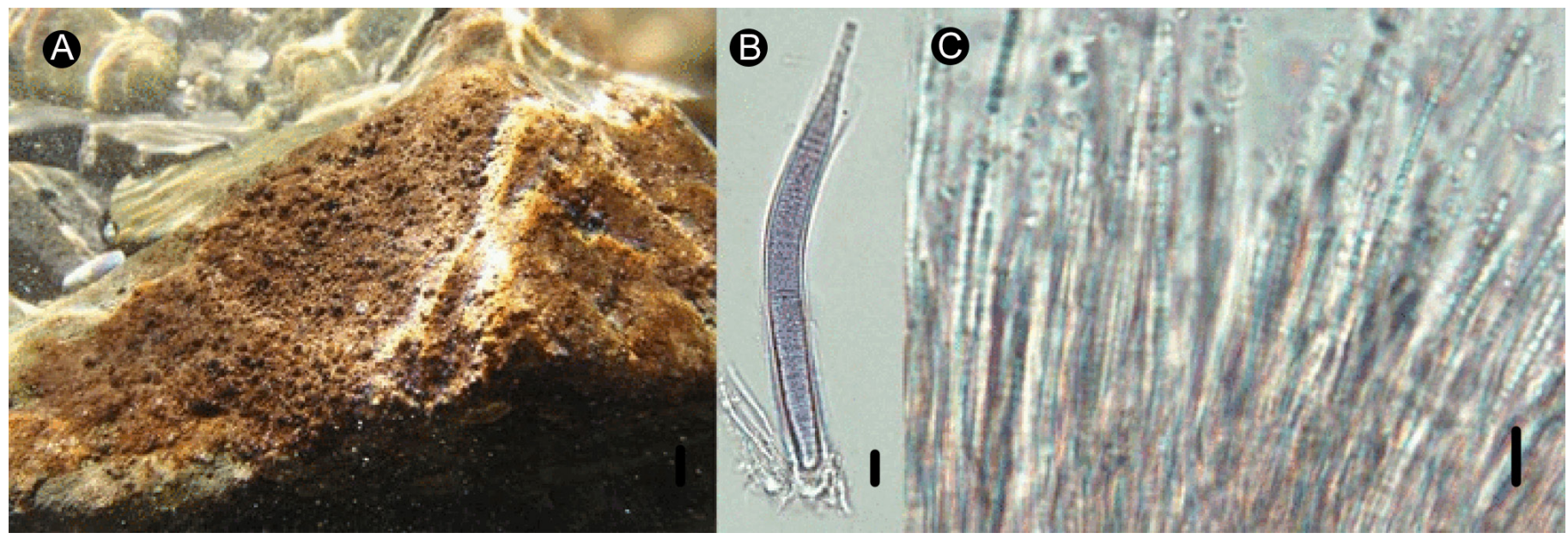

Figura 10: Heteroleibleinia fontana (Hansgirg) Anagnostidis \& Komárek. A. costra; B. filamentos alrededor de un filamento de Homoeothrix juliana (Bornet \& Flahault ex Gomont) Kirchner; C. filamentos paralelos. Escalas: $1 \mathrm{~cm}$ para la figura A; $5 \mu \mathrm{m}$ para B y C.

Hábitat: temperatura del agua $23-25{ }^{\circ} \mathrm{C} ; \mathrm{K}_{25} 1120-$ $1351 \mu \mathrm{S} \mathrm{cm}^{-1} ; \mathrm{pH}$ 7.8-8.2; velocidad de corriente 0.2-0.6 m $\mathrm{s}^{-1}$; PAR 800-1813 $\mu \mathrm{mol}$ fotón $\mathrm{m}^{-2} \mathrm{~s}^{-1}$; profundidad 0.3-0.35 $\mathrm{m}$; sustrato roca $100 \%$. Epilítica, crece entremezclada con Homoeothrix juliana.

Ejemplares examinados: MÉXICO. San Luis Potosí, municipio Aquismón, Tambaque, 24.XI.2009, 17.XI.2010, 18.XI.2011, J. Carmona y M. Cartajena PA4349 (FCME), PA4369 (FCME).

Distribución: México, San Luis Potosí; República Checa, Bohemia.

Ecología: manantiales, pozos y ríos en aguas claras y frías; formas de vida epilítica y saxícola.

Incertae sedis

Schizothrix mexicana Gomont, Ann. Sc. Nat. Bot. Ser. 7 15: 304. 1892. TIPO: MÉXICO. Huatulco. Fig.11.

Fascículos (Fig. 11A) o crecimientos esféricos (Fig. 11B); filamentos heteropolares 2-11 por fascículo (Fig. 11C), $1-8 \mathrm{~cm}$ de longitud, ramas falsas intercalares, entrelazadas, formados, 1-2 filamentos por rama apical 16-29 $\mu \mathrm{m}$ de ancho; filamentos rectos paralelos, vainas hialinas, firmes apicales (Figs. 11C, 11D) a difluente (Fig. 11E), 1.6-2.33 $\mu \mathrm{m}$ de ancho, tricomas verde o violáceos 6-9 $\mu \mathrm{m}$ ancho, no constreñidos, células vegetativas 6-7.4 $\mu \mathrm{m}$ de ancho, 4.4$5.14 \mu \mathrm{m}$ de longitud, contenido celular granuloso, sin hilera de gránulos alrededor de las paredes celulares; célula apical redondeada, no atenuada y sin caliptra, 4-10.2 $\mu \mathrm{m}$ de ancho, 5.1-11.2 $\mu \mathrm{m}$ de longitud; hormogonios (Fig. 11E) 2.3-130.5 $\mu \mathrm{m}$ de longitud, filamentos con hormogonios en su interior (Figs. 11C, 11D).

Etapas de desarrollo: el mayor crecimiento de los fascículos se registró en fín de lluvias de 2011 cuando formaron crecimientos esféricos de 6-8 cm, de consistencia suave (Fig. 11B). Filamentos jóvenes y hormogonios estuvieron presentes en todas las épocas (Figs. 11C, 11D).

Hábitat: temperatura del agua $20-26{ }^{\circ} \mathrm{C} ; \mathrm{K}_{25} 1209-$ $1476 \mu \mathrm{S} \mathrm{cm}^{-1} ; \mathrm{pH}$ 7.7-7.9; velocidad de corriente 0.17-0.36 m $\mathrm{s}^{-1}$; PAR 96-187 $\mu$ mol fotón $\mathrm{m}^{-2} \mathrm{~s}^{-1}$; profundidad 0.09-0.17 m. Epífita sobre musgo $50 \%$, saxícola sobre tronco $25 \%$ y epilítica $25 \%$, entre sus filamentos crece Phormidium subfuscum.

Ejemplares examinados: MÉXICO. San Luis Potosí, municipio Ciudad Valles, Micos, 06.II.2009, 24.XI.2009, 18.XI.2011, J. Carmona y M. Cartajena PA4345 (FCME), PA4365 (FCME).

Distribución: Ártico; Colombia; China; España; Estados Unidos de América; India; Ghana; Islas Atlánticas (Ascención, Canarias, Salvages y Solomon); Islas del Pacífico; 


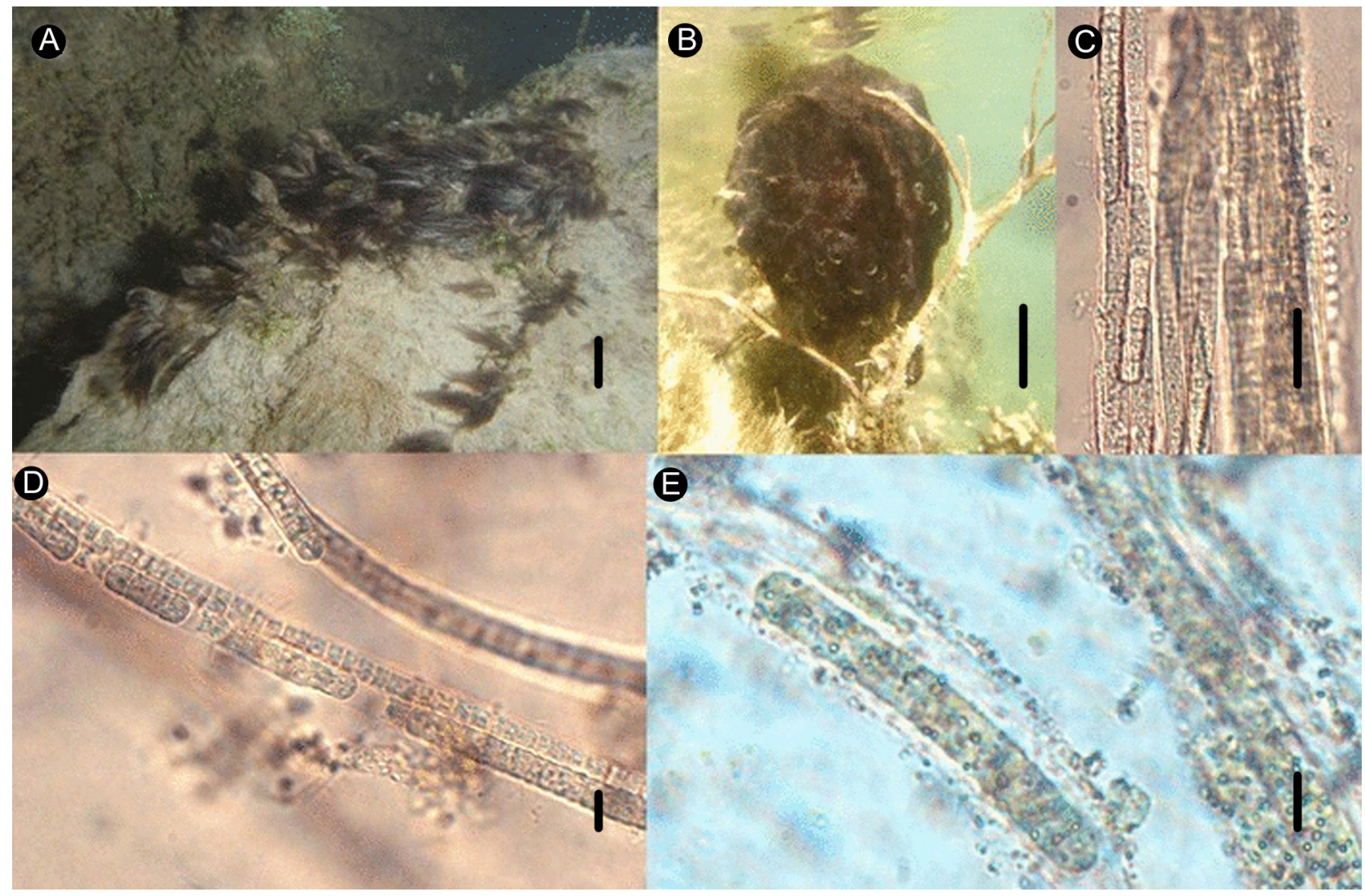

Figura 11: Schizothrix mexicana Gomont. A. fascículos; B. crecimiento esférico formado por filamentos heteropolares; C. porción basal de una rama falsa compuesta por muchos tricomas por vaina; D. porción media de una rama compuesta por un tricoma y varios hormogonios; E. hormogonio dentro de una vaina y porción de una rama con un tricoma, vaina difluente. Escalas: $3 \mathrm{~cm}$ para las figuras $A$ y B; $20 \mu \mathrm{m}$ para $C ; 8 \mu \mathrm{m}$ para $\mathrm{D}$ y $\mathrm{E}$.

Italia; Kenia; México, Guanajuato, Hidalgo, Nuevo León, Oaxaca; Micronesia; Nigeria; Oceanía; Perú; Sierra Leona; Singapur; Somalia; Tajikistan.

Ecología: arroyos, fuentes termales, manantiales y ríos; forma de vida epilítica.

\section{Formas de crecimiento de las asociaciones}

Las formas de crecimiento de las asociaciones son: colonias mucilaginosas formadas por Nostoc verrucosum, Nostoc parmelioides y Coleodesmium wrangelii (40\%), películas constituídas por Phormidium lividum y Leptolyngbya truncata (20\%), fascículos formados por Schizothrix mexicana y Phormidium subfuscum (20\%) y costras formadas por Homoeothrix juliana y Heteroleibleinia fontana (20\%) (Fig. 2, Cuadro 3). Cada asociación presentó sus especies particulares. En cuatro de los cinco ríos hubo cuatro asociaciones formadas por una especie abundante que dio estructura al crecimiento y una asociada en menor proporción (Cuadro 4). De acuerdo con los valores de abundancia relativa, Nostoc parmelioides, Nostoc verrucosum y Schizothrix mexicana fueron las especies más abundantes; Heteroleibleinia fontana, Homoeothrix juliana, Leptolyngbya truncata, Phormidium lividum y Phormidium subfuscum fueron especies comunes y Coleodesmium wrangelii, una especie rara.

\section{Caracterización ambiental}

Se reconocieron dos tipos de ríos con base en el registro de los valores promedios de temperatura, altitud, iones mayores y nutrientes (Cuadro 4, Fig. 12):

1) Ríos silíceos, Estado de México, RHA IV Balsas, con altitudes $\geq 2000 \mathrm{~m}$, valores de $\mathrm{K}_{25}$ entre $44-125 \mu \mathrm{S} \mathrm{cm}^{-1}$, temperatura del agua de $10-19{ }^{\circ} \mathrm{C}$ (Fig. 12), concentraciones iónicas de $\mathrm{Ca}^{2+}, \mathrm{SO}_{4}{ }^{3-} \mathrm{y} \mathrm{HCO}_{3}{ }^{-} \leq 64 \mathrm{mg} \mathrm{l}^{-1}$ y proporción de NID/PRS $\leq 2$ (Cuadro 4). 
Cuadro 4: Promedio y desviación estándar de parámetros físico-químicos, iones mayores y nutrientes presentes en cinco ríos con especies de cianobacterias bentónicas estudiados en la Región Hidrológica Administrativa IV (RHA IV) Balsas, México, y Región Hidrológica Administrativa IX (RHA IX) Golfo Norte, México. $\mathrm{K}_{25}=$ Conductividad específica $\left(\mu \mathrm{S} \mathrm{cm}^{-1}\right), \mathrm{HCO}_{3}=$ Bicarbonato, $\mathrm{Cl}^{-}=\mathrm{Cloro}_{1} \mathrm{SO}_{4}{ }^{3-}=$ sulfato, $\mathrm{Ca}^{2+}=\mathrm{Calcio}, \mathrm{Mg}^{2+}=\mathrm{Magnesio}$, $\mathrm{Na}^{+}=$Sodio, $\mathrm{K}^{+}=$Potasio, $\mathrm{Si}-\mathrm{SiO}_{2}=$ Sílice, $\mathrm{NH}_{4}{ }^{+}=$Amonio, $\mathrm{NO}_{2}=$ =Nitrito, $\mathrm{NO}_{3}{ }^{2-}=$ Nitrato, $\mathrm{NID}=$ Nitrógeno Inorgánico Disuelto, PRS=Fósforo Reactivo Soluble, NID/PRS=Proporción NID/PRS, nd=No determinado.

\begin{tabular}{|c|c|c|c|c|c|}
\hline \multirow{3}{*}{$\begin{array}{c}\text { Variable } \\
\text { Río }\end{array}$} & \multirow{2}{*}{\multicolumn{2}{|c|}{$\begin{array}{c}\text { RHA IV Balsas } \\
\text { Municipio Valle de Bravo }\end{array}$}} & \multicolumn{3}{|c|}{ RHA IX Golfo Norte } \\
\hline & & & & quismón y & \\
\hline & Borbollón & Carrizal & Micos & Tambaque & La Garita \\
\hline Temperatura & $15.4 \pm 2.3$ & $13.7 \pm 2.5$ & $24 \pm 3$ & $24 \pm 1$ & $22 \pm 2$ \\
\hline $\mathrm{pH}$ & $7.4 \pm 0.3$ & $7.7 \pm 0.2$ & $7.8 \pm 0.1$ & $7.8 \pm 0.4$ & $7.5 \pm 0.4$ \\
\hline $\begin{array}{l}\text { Conductividad } \\
\text { específica }\left(\mathrm{K}_{25}\right)\end{array}$ & $99 \pm 27$ & $94 \pm 23$ & $834 \pm 25$ & $1240 \pm 116$ & $1100 \pm 412$ \\
\hline $\mathrm{HCO}_{3-}^{-}$ & $64 \pm 16$ & $60 \pm 13$ & $391 \pm 15$ & $274 \pm 36$ & $2255 \pm 7$ \\
\hline $\mathrm{Cl}^{-}$ & $2.5 \pm 2$ & $1 \pm 0.3$ & $7 \pm 1$ & $6 \pm 3$ & $6 \pm 2$ \\
\hline $\mathrm{SO}_{4}^{3-}$ & $2 \pm 2$ & $1 \pm 2$ & $239 \pm 8$ & $79 \pm 3$ & $92 \pm 27$ \\
\hline $\mathrm{Ca}^{2+}$ & $11 \pm 5$ & $9 \pm 2.5$ & $341 \pm 13$ & $77 \pm 4$ & $110 \pm 66$ \\
\hline $\mathrm{Mg}^{2+}$ & $4.5 \pm 1$ & $4 \pm 1$ & $30 \pm 6$ & $18 \pm 3$ & $22 \pm 4$ \\
\hline $\mathrm{Na}^{+}$ & $6 \pm 2$ & $6 \pm 2$ & $26 \pm 3$ & $15 \pm 2$ & $12 \pm 4$ \\
\hline $\mathrm{K}^{+}$ & $2 \pm 0.2$ & $2 \pm 0.3$ & $1 \pm 0.5$ & $0.8 \pm 0.6$ & $0.7 \pm 0.4$ \\
\hline $\mathrm{Si}-\mathrm{SiO}_{2}$ & $24 \pm 8$ & $29 \pm 3$ & $7 \pm 0.8$ & $6 \pm 0.6$ & $8 \pm 0.8$ \\
\hline $\mathrm{NH}_{4}{ }^{+}$ & $4 \pm 3$ & $4 \pm 4$ & nd & $0.1 \pm 0.1$ & $0.02 \pm 0.05$ \\
\hline $\mathrm{NO}_{2-}^{-}$ & $0.1 \pm 0.1$ & $0.3 \pm 0.2$ & nd & nd & nd \\
\hline $\mathrm{NO}_{3}^{2-}$ & $5 \pm 6$ & $6 \pm 3$ & $0.8 \pm 0.2$ & $0.9 \pm 0.3$ & $0.6 \pm 0.5$ \\
\hline NID & $10 \pm 9$ & $10 \pm 5$ & $0.8 \pm 0.2$ & $0.9 \pm 0.3$ & $0.6 \pm 0.5$ \\
\hline PRS & $1 \pm 0.4$ & $1 \pm 1$ & $0.04 \pm 0.03$ & $0.4 \pm 0.3$ & $0.1 \pm 0.1$ \\
\hline NID/PRS & $1.2 \pm 0.7$ & $2 \pm 1$ & $39 \pm 40$ & $4 \pm 5$ & $6 \pm 1$ \\
\hline
\end{tabular}

2) Ríos calcáreos, San Luis Potosí, RHA IX Golfo Norte, con altitudes $\leq 150 \mathrm{~m}$, valores de $\mathrm{K}_{25}$ entre $515-1476 \mu \mathrm{S} \mathrm{cm}^{-1}$, temperatura del agua de $20-26^{\circ} \mathrm{C}$ (Fig. 12), concentraciones iónicas de $\mathrm{Ca}^{2+}, \mathrm{SO}_{4}{ }^{3-}$ y $\mathrm{HCO}_{3} \leq 341 \mathrm{mg} \mathrm{l}^{-1}$ y proporción de NID/PRS $\leq 39$ (Cuadro 4).

De acuerdo con las concentraciones de NID y PRS los ríos silíceos Borbollón y Carrizal tuvieron condiciones mesotróficas de NID (0.9-1 $\left.\mathrm{mg} \mathrm{l}^{-1}\right)$ y eutróficas de PRS (1 $\left.\mathrm{mg} \mathrm{l}^{-1}\right)$, mientras que los ríos calcáreos La Garita, Micos y Tambaque fueron mesotróficos con respecto al NID (0.2-1.3 $\left.\mathrm{mg} \mathrm{l}^{-1}\right)$; los ríos La Garita y Micos fueron oligotróficos al fósforo (0.01$\left.0.1 \mathrm{mg}^{-1}\right)$ y río Tambaque, mesotrófico-eutrófico con respecto al fósforo (0.12-0.7 $\mathrm{mg} \mathrm{l}^{-1}$ ) (Cuadro 4).

Con respecto a la caracterización local, los cinco ríos mostraron diferencias. En el Estado de México, RHA IV Balsas, río Borbollón es un cauce sin pendiente con sombreado intermedio por la vegetación de ribera, profundidad intermedia e intervalos de intermedios a altos de velocidad de corriente, con rocas y cantos rodados sedimentarios. En contraste, en río Carrizal hay una pendiente aproximada de $30^{\circ}$, iluminación directa, profundidad baja e intervalos de intermedios a altos de velocidad de corriente, con rocas metamórficas mayores a un metro de diámetro con una parte expuesta a la atmósfera.

En la RHA IX Golfo Norte, municipio Ciudad Valles, Micos es el río de mayor orden y heterogeneidad, en la zona donde crecieron Schizothrix mexicana y Phormidium subfuscum el terreno es irregular con muchos recovecos, la vegetación de ribera alta y la iluminación baja, existe una poza de $80 \mathrm{~cm}$ de profundidad, la velocidad de corriente es baja y el sustrato estuvo compuesto por rocas sumergidas cubiertas de carbonato de calcio. Río Tambaque es homogéneo, el terreno es plano, sin pendiente, la incidencia de luz es alta y directa, la profundidad regular, la velocidad de corriente intermedia, con cantos rodados y rocas sedimentarias. Por último, río La Garita es también un terreno sin 
Temperatura

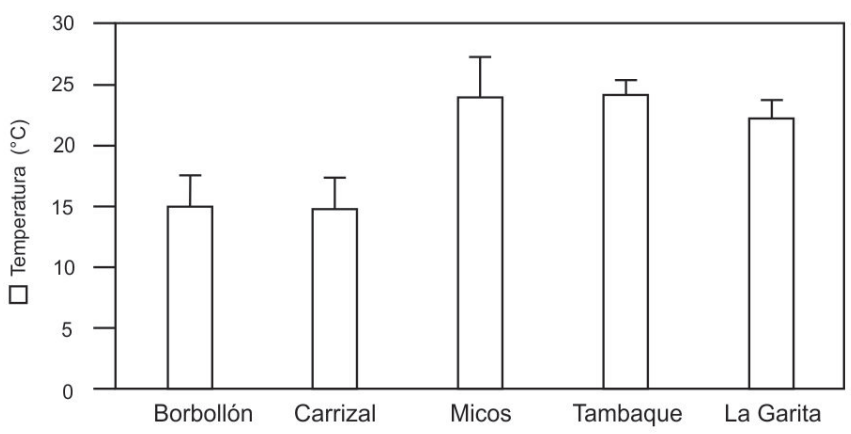

Nutrientes

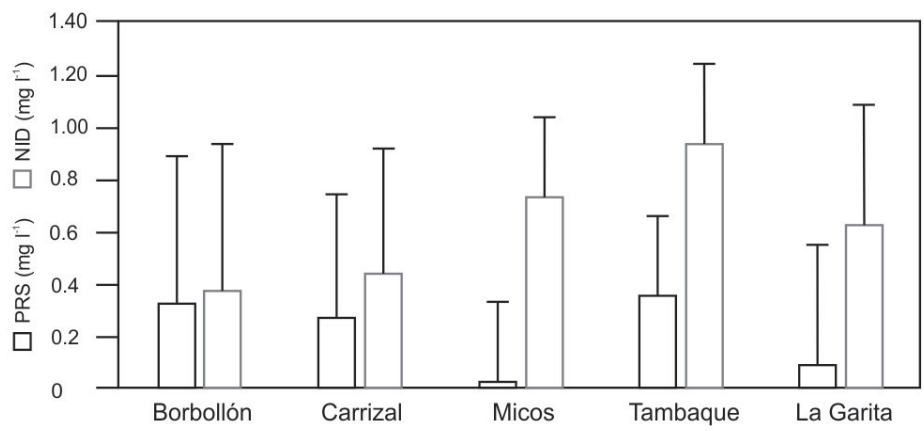

Figura 12: Promedio y desviación estándar de la temperatura del agua y presencia de nutrientes en los ríos silíceos Borbollón y Carrizal, Estado de México, RHA IV Balsas, México, y en los tres ríos calcáreos La Garita, Micos y Tambaque, San Luis Potosí, RHA IX, Golfo Norte, México. NID=Nitrógeno Inorgánico Disuelto; PRS=Fósforo Reactivo Soluble; $\mathrm{mg} \mathrm{l}^{-1}=$ miligramos por litro.

pendiente, pero con una zona de cantos rodados en la orilla que queda expuesta en época de secas. En la zona donde crece Nostoc verrucosum, la cobertura vegetal es alta y la iluminación muy baja, con velocidad de corriente alta. El sustrato es travertino y con rocas sumergidas.

\section{Caracterización microambiental}

De acuerdo con los test de Kruskal-Wallis y Mann-Whitney, se encontraron diferencias significativas entre asociaciones con los factores microambientales: velocidad de corriente $(U=82, p \leq 0.05)$, profundidad $(U=51, p<0.05)$ y luz fotosintéticamente activa $(U=316, p<0.05)$ (Fig. 13).

Las asociaciones de Schizothrixmexicana-Phormidium subfuscum, Homoeothrix juliana-Heteroleibleinia fontana y Nostoc parmelioides-Coleodesmiun wrangelii estuvieron en zonas con velocidad de corriente nula a moderada (promedio $<0.4 \mathrm{~m} \mathrm{~s}^{-1}$ ), Phormidium lividum-Leptolyngbya truncata en velocidad de corriente intermedia (promedio $=0.5 \mathrm{~m} \mathrm{~s}^{-1}$ ) y Nostoc verrucosum en velocidad de corriente alta (promedio $>0.7 \mathrm{~m} \mathrm{~s}^{-1}$ ).

Con base en la profundidad, Nostoc parmelioidesColeodesmiun wrangelii se registraron en zonas someras a subaéreas (promedio $<0.04 \mathrm{~m}$ ); Phormidium lividumLeptolyngbya truncata, Schizothrix mexicana-Phormidium subfuscum y Nostoc verrucosum en profundidad intermedia (promedio $<0.17 \mathrm{~m}$ ) y Homoeothrix julianaHeteroleibleinia fontana en zonas profundas (promedio=0.33 m).
Se reconocieron tres grupos de especies con base en la cantidad de PAR: 1) Phormidium lividum-Leptolyngbya truncata, Schizothrix mexicana-Phormidium subfuscum y Nostoc verrucosum en sitios con bajos valores de PAR (40 $\mu \mathrm{mol}$ fotones $\left.\mathrm{m}^{2} \mathrm{~s}^{-1}\right)$; 2) Nostoc parmelioides-C. wrangelii con valores de radiación intermedia (promedio $=464 \mu \mathrm{mol}$ fotones $\mathrm{m}^{2} \mathrm{~s}^{-1}$ ) y 3) Homoeothrix juliana-Heteroleibleinia fontana en los sitios con PAR más alta (promedio=1130 $\mu \mathrm{mol}$ fotones $\left.\mathrm{m}^{2} \mathrm{~s}^{-1}\right)$. Cada asociación se estableció en sustratos particulares en tamaño y disposición siendo las rocas y cantos rodados los más frecuentes.

\section{Distribución}

Ocurren dos patrones de distribución espacial en las especies de cianobacterias bentónicas en los ríos de la región central de México: las especies de ríos silíceos en los ríos Borbollón y Carrizal, y las especies de ríos calcáreos en los ríos La Garita, Micos y Tambaque.

La relación entre la distribución de las especies y los factores ambientales se evaluó con un análisis de redundancia (RDA); este test relacionó las variaciones estacionales de los parámetros ambientales y los porcentajes de cobertura de las asociaciones y pudo explicar $80 \%$ de la variación de la abundancia en los dos primeros ejes ( $p \leq 0.05$, Fig. 14). El eje uno explicó $48 \%$ de la varianza y mostró una correlación positiva entre los ríos calcáreos con la temperatura del agua, la proporción de NID/PRS y las especies Schizothrix mexicana-Phormidium subfuscum, 

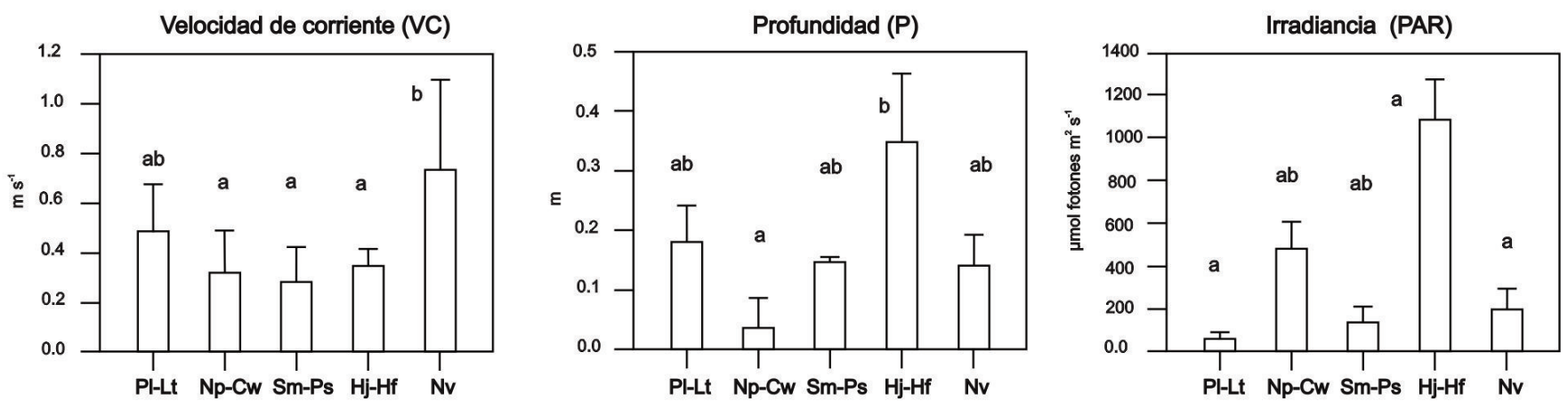

Figura 13: Promedio y desviación estándar de las variables microambientales presentes en dos ríos silíceos (Borbollón y Carrizal), Estado de México, RHA IV Balsas, México y tres ríos calcáreos (La Garita, Micos y Tambaque), San Luis Potosí, RHA IX Golfo Norte, México. Velocidad de corriente=VC, radiación fotosintéticamente activa=PAR; profundidad=P. Las especies que comparten letras es porque no hubo diferencias significativas. Las letras distintas ( $\mathrm{a}, \mathrm{b} \circ \mathrm{ab}$ ) mostraron diferencias significativas entre especies de acuerdo con el test de Mann-Whitney ( $\mathrm{p}=0.05)$. $\mathrm{Pl}=P$ hormidium lividum (Hansgirg) Forti; Lt=Leptolyngbya truncata (Lemmermann) Anagnostidis \& Komárek; Np=Nostoc parmelioides Kützing ex Bornet \& Flahault; $\mathrm{CW}=$ Coleodesmium wrangelii Borzì ex Geitler; Sm=Schizothrix mexicana Gomont; Ps=Phormidium subfuscum Kützing ex Gomont; Hj=Homoeothrix juliana (Bornet \& Flahault ex Gomont) Kirchner; Hf=Heteroleiblenia fontana (Hansgirg) Anagnostidis \& Komárek; Nv=Nostoc verrucosum Vaucher ex Bornet \& Flahault.

Nostoc verrucosum y Homoeothrix juliana-Heteroleibleinia fontana. El eje dos explicó $32 \%$ de variación y mostró correlación positiva entre fósforo reactivo soluble (PRS) y las especies de ríos silíceos Phormidium lividum-Leptolyngbya truncata y Nostoc parmelioides-Coleodesmium wrangelii.

\section{Discusión}

Existieron dos grupos de especies, el de ríos silíceos de montaña en la RHA IV Balsas y el de los ríos calcáreos tropicales en la RHA IX Golfo Norte. Las cianobacterias de los ríos silíceos de los géneros Phormidium Kützing ex Gomont, Leptolyngbya Anagnostidis \& Komárek, Nostoc Vaucher ex Borntet \& Flahault y Coleodesmium Borzì ex Geitler concuerdan con los registros de estas especies en ríos de regiones templadas (Kann y Komárek, 1970; John et al., 2003; Komárek y Anagnostidis, 2005; Komárek, 2013), mientras que Nostoc verrucosum, Homoeothrix juliana y Phormidium subfuscum han sido reportadas para ríos calcáreos españoles (Aboal et al., 2002; Jubera et al., 2009).

La presencia de las especies distintas y la heterogeneidad de cada río permiten caracterizar los microambientes resultado de las diferencias en variables locales, como el porcentaje de sombreado por la vegetación de ribera, el tamaño y disposición del sustrato y el patrón de flujo de agua (Zanini et al., 2009; Branco et al., 2014; RodríguezFlores y Carmona, 2018).

Con respecto a la estructura de las asociaciones, las colonias mucilaginosas, los fascículos, las películas y las costras proveen protección contra el arrastre y mantienen un ambiente rico en nutrientes alrededor de sus superficies, lo cual favorece la mayor producción de biomasa (McCormick y Stevenson, 1991). Además, las especies macroscópicas abundantes favorecen la colonización de las especies asociadas registradas y es probable que entre ellas exista una interacción positiva (Burkholder, 1996; Mollenhauer et al., 1999). Las asociaciones, en coincidencia con los factores microambientales, permitieron caracterizar los diferentes microambientes donde se establecen las cianobacterias bentónicas en ríos, lo cual corresponde con lo descrito por Carmona y Montejano (1993).

Con base en nuestros resultados proponemos que las colonias mucilaginosas de Nostoc parmelioidesColeodesmium wrangelii y Nostoc verrucosum estuvieron presentes en zonas estresantes en términos de luz y velocidad de corriente. El mucílago denso de las colonias mucilaginosas compactas de Nostoc verrucosum reduce la fuerza de abrasión, en tanto que los acinetos y heterocitos permanecen latentes en época de secas (Krupek et al., 


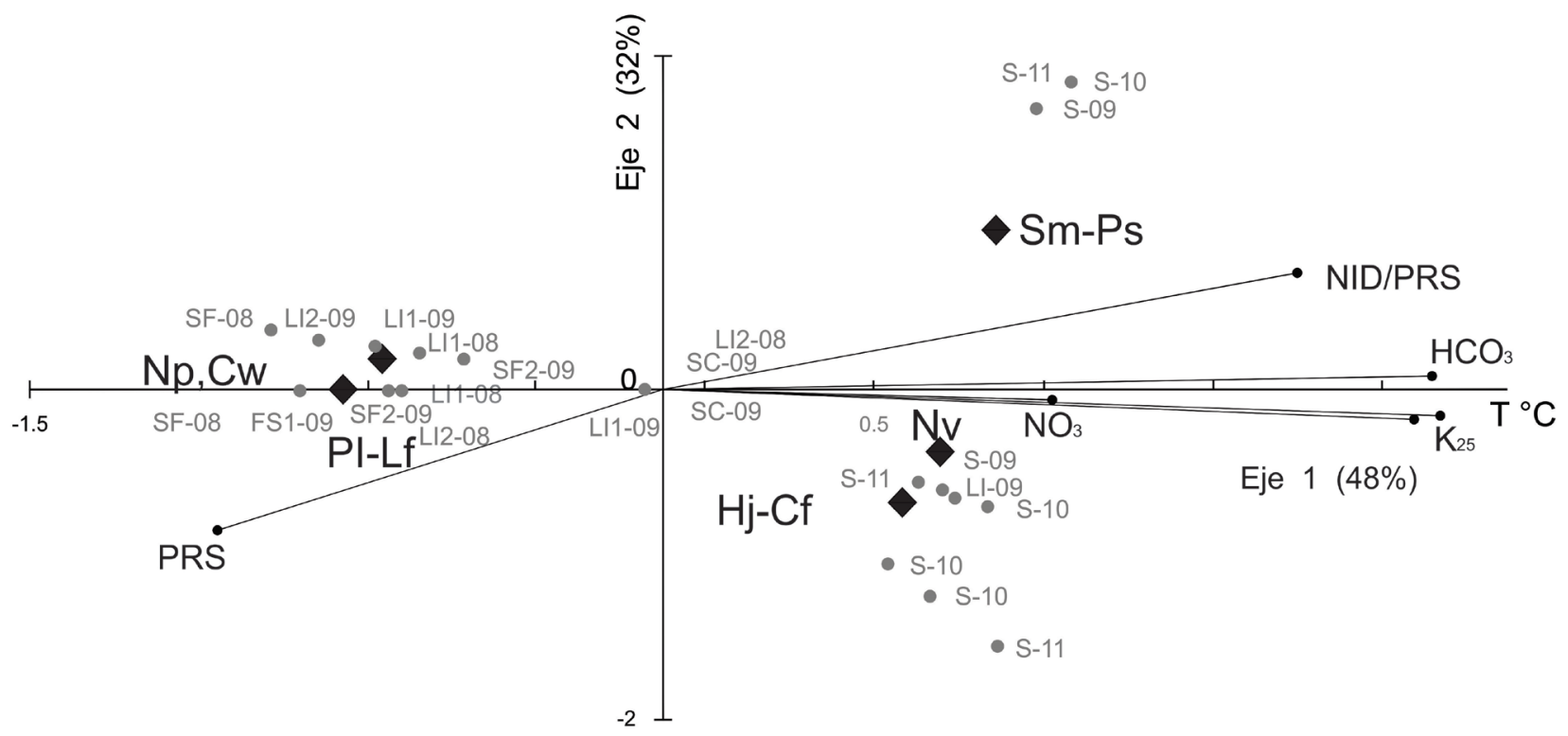

Figura 14: Análisis de redundancia de la distribución de las asociaciones de cianobacterias en función de los datos físico-químicos registrados en dos ríos silíceos, Estado de México, RHA IV Balsas, México y tres ríos calcáreos, San Luis Potosí, RHA IX Golfo Norte, México. Bicarbonato=HCO - ; Nitrógeno inorgánico disuelto=NID; Fósforo reactivo soluble=PRS. Épocas: Lluvia (LI), secas frías (SF), secas cálidas (SC) y secas (S). El número seguido a la abreviatura de secas o lluvias se refiere al número de recolecta por época. Los números 08, 09, 10 y 11 hacen referencia a los años 2008,2009 , 2010 y 2011, respectivamente. Pl=Phormidium lividum (Hansgirg) Forti; Lt=Leptolyngbya truncata (Lemmermann) Anagnostidis \& Komárek; Np=Nostoc parmelioides Kützing ex Bornet \& Flahault; $\mathrm{CW}=$ Coleodesmium wrangelii Borzì ex Geitler; Sm=Schizothrix mexicana Gomont; Ps=Phormidium subfuscum Kützing ex Gomont; Hj=Homoeothrix juliana (Bornet \& Flahault ex Gomont) Kirchner; Hf=Heteroleiblenia fontana (Hansgirg) Anagnostidis \& Komárek; Nv=Nostoc verrucosum Vaucher ex Bornet \& Flahault.

2012). Las películas de Phormidium lividum-Leptolyngbya truncata se desarrollaron en sitios con valores intermedios de velocidad de corriente al disminuir la fricción; sus vainas representan superficies donde el agua se puede deslizar. En relación con el recurso luz, las especies estudiadas podrían considerarse adaptadas a sitios con baja irradiancia donde la vegetación de ribera redujó la cantidad de luz disponible. La excepción a lo anterior ocurre con Homoeothrix julianaHeteroleibleinia fontana, las cuales formaron costras en zonas abiertas con valores altos de PAR. Los iones presentes sobre las vainas de sus filamentos pueden ser un mecanismo de protección contra la luz ultravioleta (Beltrán-Magos et al., 2013).

Por otro lado, la presencia estacional recurrente de las mismas especies de cianobacterias puede ser resultado de la producción de hormogonios y la germinación de acinetos los cuales resisten condiciones ambientales estre- santes (Potts, 1999) en la época de secas e inicio de lluvias.

La taxonomía de las cianobacterias puede abordarse a partir de la propuesta del Código Internacional de Nomenclatura de Procariontes (Parker et al., 2019) o con el Código Internacional de Nomenclatura de Algas, Hongos y Plantas (Turland et al., 2018). En los últimos años se ha incrementado el número de taxa definidos molecularmente. Sin embargo, la importancia de los caracteres morfológicos y datos ecológicos se refleja en el alto número de secuencias disponibles en la base de datos de la página del Centro Nacional para la Información de Biotecnología (NCBI) identificadas sólo a nivel genérico. Muchas secuencias no cuentan con una buena descripción del hábitat donde se desarrollan, ni una buena caracterización morfológica de las poblaciones naturales. Por ejemplo, de las primeras 492 secuencias disponibles en la base de datos de NCBI del género Phormidium Kützing ex Gomont, 207 están sólo a 
nivel género, 85 están etiquetadas a nivel genérico como de material no cultivable, 65 corresponden a Phormidium ambiguum Gomont y las 135 restantes corresponden a sólo cuatro especies de este género (NCBI, 2020). La carencia de esta información se hace evidente en la delimitación de taxa con base en su presencia en hábitats con rangos ecológicos definidos y estrechos (Anagnostidis y Komárek, 1988; Carmona et al., 2005; Fiore et al., 2007; Gold-Morgan et al., 2015; Martins y Branco, 2016; Becerra-Absalón et al., 2018; Akagha et al., 2019; Cai et al., 2019a, b).

De acuerdo con el grado de caracterización taxonómica hecho en el presente estudio, las especies Phormidium lividum, Leptolyngbya truncata, Schizothrix mexicana y Heteroleibleinia fontana con poca información morfológica y ecológica y sin secuencias en el GenBank, podrían tratarse de nuevos taxa no descritos para ríos de regiones tropicales. Especies crípticas han sido descritas para varios géneros y/o especies de cianoprocariontes (Cassamatta et al., 2003; Engene et al., 2012, 2013, 2018; McGregor y Sendall, 2015). De acuerdo con lo anterior, es posible que los registros de Nostoc parmelioides, Nostoc verrucosum Coleodesmium wrangelii, Phormidium subfuscum y Homoeothrix juliana descritos en este trabajo sean especies crípticas en regiones tropicales, similares morfológicamente a las especies descritas para ríos templados. Este proceso de especiación molecular podría ser común para otras especies, de amplia distribución en la región central de México como Blennothrix ganeshii M. Watanabe \& Komárek (Carmona et al., 2005) y Placoma regulare P.A. Broady \& M. Ingerfeld (Rodríguez-Flores y Carmona, 2018).

El presente estudio no abarcó caracteres ultraestructurales ni moleculares, pero está planteado con el concepto de especie como un grupo de poblaciones (cepas) con el mismo genotipo, un fenotipo estable, con características definidas y reconocibles con diferentes límites de variación y que comparten las mismas demandas ecológicas las cuales ocurren reiteradamente en tiempo y localidades (Komárek, 2016). En este trabajo se definieron y reconocieron especies con base en caracteres fenotípicos estables, su delimitación ecológica y su recurrencia espacial y temporal. Esta aportación es relevante porque constituye la base de futuros estudios sistemáticos y ecológicos.
Las descripciones morfológicas y la caracterización ambiental de los taxa de cianoprocariontes bentónicos en zonas tropicales son la base y punto de referencia para la caracterización polifásica que incluya herramientas moleculares y ultraestructurales a realizar en estudios posteriores. Los patrones similares de distribución restringida, la baja diversidad por sitio, la organización en asociaciones y la permanencia anual de las especies contribuyen al conocimiento de la biodiversitad y son la base para proyectos enfocados en el manejo e impacto del cambio climático sobre los ríos de la región central de México.

\section{Contribución de autores}

JCJ, EPU y MGCA llevaron a cabo el diseño del proyecto y el trabajo de campo; MGCA observó las muestras, realizó las determinaciones taxonómicas, el análisis estadístico, la interpretación de datos y la discusión de los resultados; MGCA y JCJ redactaron el escrito. JCJ y MGCA editaron las figuras. EPU realizó la revisión crítica del escrito.

\section{Financiamiento}

El Posgrado del Instituto de Ciencias del Mar y Limnología a través del Consejo Nacional de Ciencia y Tecnología otorgó la beca doctoral 35164 a la primera autora. El Programa para los Proyectos de Investigación e Innovación Tecnológica (PAPIIT) de la Universidad Nacional Autónoma de México (UNAM) (proyecto IN220115) y la Agencia Española de Cooperación Internacional (proyectos A/010529/07 y A/016417/08), Banco Santander/Agencia Española de Cooperación con Iberoamérica-Universidad Autónoma de Madrid financiaron las salidas al campo.

\section{Agradecimientos}

Se agradece al hidrobiólogo Sergio Castillo del Instituto de Ciencias del Mar y Limnología (ICMyL) de la Universidad Nacional Autónoma de México (UNAM), por el análisis de los nutrientes; a Alejandra Aguayo, N. Ceniceros y O. Cruz del Instituto de Geofísica de la UNAM, por el análisis de los iones mayores; a Yenny Beltrán, Miriam Bojorge y Rocío Ramírez por la ayuda en el trabajo de campo, a Verónica Aguilar por la elaboración del mapa y a Rogelio Rodríguez por la asesoría para el análisis estadístico de redundancia, RDA. 


\section{Literatura citada}

Aboal, M. 1988. Aportación al conocimiento de las algas epicontinentales del sudeste de España. III: Cianofíceas (Cyanophyceae Schaffner 1909). Anales del Jardín Botánico de Madrid 45: 1-46.

Aboal, M., M. A. Puig, P. Mateo y E. Perona. 2002. Implications of cyanophyte toxicity on biological monitoring of calcareous streams in north-east Spain. Journal of Applied Phycology 14: 49-56. DOI: https://doi.org/10.1023/A:1015298905510

Addinsoft. 2013. XLSTAT for Windows, versión 2015.1. New York, USA. https://www.xlstat.com/en/ (consultado junio de 2015).

Akagha, C. S., R. J. Johansen, I. D. Nwankwo y K. Yin. 2019. Lagosinema tenuis gen. et sp. nov. (Prochlorotricaceae, Cyanobacteria): a new brackish water genus from Tropical Afrika. Fottea, Olomouc 19(1): 1-12. DOI: https://doi. org/10.5507/fot.2018.012

Allan, J. D. y M. Castillo. 2007. Stream Ecology. Structure and function of running waters. Springer. Dordrecht, Netherlands. 441 pp. DOI: https://doi.org/10.1007/978-14020-5583-6

Anagnostidis, K. y J. Komárek. 1988. Modern approach to the classification systems of cyanophytes. 3-Oscillatoriales. Archiv für Hydrobiologie Supplement-Algological Studies 50-53: 327-472.

Becerra-Absalón, I., B. Rodarte, K. Osorio, L. Alba-Lois, C. SegalKischinevzky y G. Montejano. 2013. A new species of Brasilonema (Scytonemataceae, Cyanoprokaryota) from Tolantongo, Hidalgo, Central Mexico. Fottea, Olomouc 13(1): 25-38. DOI: https://doi.org/10.5507/fot.2013.003

Becerra-Absalón, I., J. R. Johansen, M. A. Muñoz-Martín y G. Montejano. 2018. Chroakolemma gen. nov. (Leptolyngbyaceae, Cyanobacteria) from soil biocrust in the semi-desert Central Region of Mexico. Phytotaxa 367(3): 201-218. DOI: https://doi.org/10.11646/ phytotaxa.367.3.1

Beltrán-Magos, Y., J. Carmona, G. Vilaclara y M. Bojorge-García. 2013. Calcification of the filamentous cyanobacterium Blennothrix ganeshii in calcareous tropical streams of central Mexico region. Hidrobiológica 23(1): 17-27.

Betancourt, G. V. 2018. Caracterización estructural y funcional de comunidades microscópicas fotosintéticas (aerobias) formadoras de microbialitas en lagos de origen volcánico.
Lago Atexcac, México. Maestría en Ciencias. Instituto de Ciencias del Mar y Limnología, Universidad Nacional Autónoma de México. Cd. Mx., México. 101 pp.

Bojorge-García, M., J. Carmona, Y. Beltrán y M. Cartajena. 2010. Temporal and spatial distribution of macroalgal communities of mountain streams in Valle de Bravo Basin, central Mexico. Hydrobiologia 641(1): 159-169. DOI: https://doi.org/10.1007/s10750-009-0074-5

Branco, L. H., J. O. Necchi y C. C. Branco. 2001. Ecological distribution of Cyanophyceae in lotic ecosystems of Sao Paulo. Revista Brasileira de Botanica 24(1): 99-108. DOI: https://dx.doi.org/10.1590/S0100-84042001000100011

Branco, C. C., C. P. Bispo, C. K. Peres, A. F. Tonetto y L. H. Branco. 2014. The roles of environmental conditions in controlling stream macroalgal communities. Hydrobiologia 732: 123 132. DOI: https://dx.doi.org/10.1007/s10750-014-1852-2

Burkholder, J. M. 1996. Interactions of benthic algae with their substrata. In: Stevenson, R., L. Bothwell y R. Lowe (eds.). Algal ecology: Freshwater benthic ecosystems. Academic Press. California, USA. Pp. 253-297.

Cai, F., X. Li, Y. Yang, N. Jia, D. Huo y R. Li. 2019a. Compactonostoc shennnongijaensis gen. y sp. nov. (Nostocales, Cyanobacteria) from a wet rocky wall in China. Phycologia 58(2): 200-210. DOI: http://doi.org/10.1080/00318884.201 8.1541270

Cai, F., X. Li, R. Geng, X. Peng y R. Li. 2019b. Phylogenetically distant clade of Nostoc-like taxa with the description of Minunostoc gen. nov. and Minunostoc cylindricum sp. nov. Fottea, Olomouc 19(1): 13-24. DOI: http://dx.doi. org/10.5507/fot.2018.013

Carmona, J. y G. Montejano. 1993. Caracterización ficológica en manantiales de la Cuenca baja del sistema hidrológico del Pánuco, México. Boletín de la Sociedad Botánica de México 53: 21-41. DOI: https://doi.org/10.17129/botsci.1414

Carmona, J., Y. Beltrán y L. Collado-Vides. 2005. Taxonomy and distribution of freshwater Blennothrix ganeshii Watanabe et Komárek (Oscillatoriaceae. Cyanophyceae) from central Mexico. Nova Hedwigia 80(3-4): 323-333. DOI: https://doi. org/10.1127/1864-1318/2005/0117-0133

Cartajena, A. M. 2004. Taxonomía y distribución de Cianofitas filamentosas de La Huasteca, México. Tesis de licenciatura. Facultad de Ciencias, Universidad Nacional Autónoma de México. México, D.F., México. 211 pp. 
Cassamata, D. A., M. L. Vis y R. G. Sheath. 2003. Cryptic species in cyanobacterial systematics: a case study of Phormidium retzii (Oscillatoriales) using RAPD molecular markers and 16S rDNA sequence data. Aquatic Botany 77: 295-309. DOI: https://doi.org/10.1016/j.aquabot.2003.08.005

CICESE. 2020. Base de datos climatológica nacional (CLICOM). Centro de Investigación Científica de Educación Superior de Ensenada (CICESE). Ensenada, México.

CONAGUA. 2010. Estadísticas del agua en México. Comisión Nacional del Agua. México, D.F., México. 258 pp.

CONAGUA. 2018. Estadísticas del agua en México. Comisión Nacional del Agua. Cd. Mx., México. 306 pp.

Dodds, W. K., J. R. Jonesy E. B. Welch. 1998. Suggested classification of stream trophic state: distributions of temperate stream types by chlorophyll, total nitrogen and phosphorus. Water Research 32(5): 1455-1462.

Douterelo, I., E. Perona y P. Mateo. 2004. Use of cyanobacteria to assess water quality in running waters. Environmental Pollution 127: 377-384. DOI: https://doi.org/10.1016/j.env. pol.2003.08.016

Engene, N., E. C. Rottacker, H. Choi, T. Byrum, J. H. Kaštovský, J. Komárek y W. H. Gerwick. 2012. Moorea producens gen. nov., sp. nov and Moorea bouillonii comb. nov., tropical marine cyanobacteria rich in bioactive secondary metabolites. International Journal of Systematic Evolutionary Microbiology 62(5): 1172-1179. DOI: https:// doi.org/10.1099/ijs.0.033761-0

Engene, N., T. Byrum, A. Thor, M. H. Ellisman, W. H. Gerwick y V. J. Paul. 2013. Five chemically rich species of tropical marine cyanobacteria of the genus Okeania gen. nov. (Oscillatoriales, cyanobacteria). Journal of Phycology 49(6): 1095-1106. DOI: https://doi.org/10.1111/jpy.12115

Engene, N., A. Tronholm y V. J. Paul. 2018. Uncovering cryptic diversity of Lyngbya: The new tropical marine cianobacterial genus Dapis (Oscillatoriales). Journal of Phycology 54: 435466. DOI: https://doi.org/10.1111/jpy.12752

Fiore, M. F., C. L. Sant'Anna, M. T. de Paiva Azevedo, J. Komárek, J. Kaštovský, J. Sulek y A. Sturion Lorenzi. 2007. The cyanobacterial genus Brasilonema gen. nov., a molecular and phenotypic evaluation. Journal of Phycology 43: 789-798. DOI: https://doi.org/10.1111/j.1529-8817.2007.00376.x

Geitler, L. 1932. Cyanophyceae. Akademische Verlagsgesellschaft. Leipzig, Deutschland. 1196 pp.
Gold-Morgan, M., L. González, H. León y G. Montejano. 2015. Coccoid cyanoprokaryote Nisada stipitata morphogen. et. sp. nov. from the supralittoral zone in the tropical Mexican Pacific. Phytotaxa 220(3): 268-276. DOI: https://dx.doi. org/10.11646/phytotaxa.220.3.5

Guiry M. y G. Guiry. 2020. AlgaeBase World-Wide electronic publication. National University of Ireland. Galway, Ireland. http://www.algaebase.org (consultado noviembre de 2019).

Hrouzek, P., A. Lukesova, J. Mares y S. Ventura. 2013. Description of the cyanobacterial genus Desmonostoc gen. nov. including D. muscorum comb. nov. as a distinct, phylogenetically coherent taxon related to the genus Nostoc. Fottea 13(2): 201-213. DOI: https://doi.org/10.5507/fot.2013.016

Ibarra, G. C. 2017. Caracterización morfológica de los cianoprocariontes y las algas epilíticas de la zona arqueológica de Yaxchilán, Chiapas. Maestría en Ciencias Biológicas. Facultad de Ciencias, Universidad Nacional Autónoma de México. Cd. Mx., México 135 pp.

John, D. M., B. A. Whitton y A. J. Brook (eds.). 2003. The Freshwater Algal Flora of the British Isles. An Identification Guide to Freshwater and Terrestrial Algae. Cambridge University Press. Cambridge, UK. 702 pp.

Jubera, E., M. Fernández, B. Estébanez y Jara Vassal’lo. 2009. Comunidades de cianobacterias bentónicas en el enclave de El Pozo Azul (Karst de Burgos). In: Llamas, F. y C. Acedo (eds.). Botánica Pirenaico-Cantábrica en el siglo XXI. Universidad de León. León, España. 739 pp.

Kann, E. V. y J. Komárek. 1970. Systematische-Ökologische Bemerkungen zu den Arten des Formenkreises Phormidium autumnale. Hydrologie 32(2): 495-518.

Komárek, J. 2013. Cyanoprokaryota Part 3: Heterocytous genera. Süßwasserflora von Mitteleuropa 19(3): 1-1131.

Komárek, J. 2016. A polyphasic approach for the taxonomy of cyanobacteria: principles and applications. European Journal of Phycology 513: 346-353. DOI: https://doi.org/10 .1080/09670262.2016.1163738

Komárek, J. y K. Anagnostidis. 2005. Cyanoprokaryota Part 2: Oscillatoriales. Süßwasserflora von Mitteleuropa 19(2): 1-759.

Komárek, J., J. Kaštovský, J. Mareš y J. Johansen. 2014. Taxonomic classification of cyanoprokaryotes (cyanobacterial genera) 2014, using a polyphasic approach. Preslia 86: 295-335. 
Krupek, R. A., C. C. Branco y C. K. Peres. 2012. Spatial variations at different observational scales and the seasonal distributions of stream macroalgae in a Brazilian subtropical region. Brazilian Journal of Botany 35(3): 249-257.

Loyo, E. E. 2015. Ecología de algas epilíticas de la zona arqueológica, Chiapas. Maestría en Ciencias Biológicas. Facultad de Ciencias, Universidad Nacional Autónoma de México. Cd. Mx., México. 155 pp.

Maceda, R., R. Tavera y E. Novelo E. 2017. Ecología de cianoprocariontes epifitas que habitan ambientes lóticos de la Cantera Oriente, Ciudad de México. Hydrobiológica 27(3): 327-336.

Margalef, R. 1983. Limnología. Omega. Barcelona, España. 1014 pp.

Martins, M. D. y L. H. Branco. 2016. Potamolinea gen. nov. (Oscillatoriales, Cyanobacteria): a phylogenetically and ecologically coherent cyanobacterial genus. International Journal of Systematics and Evolutionary Microbiology 66: 3632-3641. DOI: http://doi.org/10.1099/ijsem.0.001243

McCormick, P. V. y R. J. Stevenson. 1991. Mechanisms of benthic algal succession in lotic environments. Ecology 72: 18351848. DOI: https://doi.org/10.2307/1940982

McGregor, G. B. y B. C. Sendall. 2015. Phylogeny and toxicology of Lyngbya wollei (Cyanobacteria/Oscillatoriales) form northeastern Australia, with a description of Microseira gen. nov. Journal of Phycology 51: 109-119. DOI: https://doi. org/10.1111/jpy.12256

Meave del Castillo, M., M. E. Zamudio-Resendiz y M. CastilloRivera. 2012. Riqueza fitoplanctónica de la bahía de Acapulco y zona costera aledaña, Guerrero, México. Acta Botanica Mexicana 100: 405-487. DOI: https://doi. org/10.21829/abm100.2012.41

Miranda, G. A. 2015. Estimación de la precipitación en la cuenca del río Balsas mediante el uso de satélites. Tesis de licenciatura. Facultad de Ingeniería, Universidad Nacional Autónoma de México. México, D.F., México. 79 pp.

Mollenhauer, D., R. Bengtsson y E. Lindstrøm. 1999. Macroscopic cyanobacteria of the genus Nostoc: a neglected and endangered constituent of European inland aquatic biodiversity. European Journal of Phycology 34(04): 349360. DOI: https://doi.org/10.1080/09670269910001736412

Montejano, G., J. Carmona y E. Cantoral. 2000. Algal communities from calcareous springs and streams from central Mexico:
A synthesis. In: Munawar, S. G., I. Lawrence, F. Munawar y D. F. Malley (eds.). Aquatic Ecosystems of Mexico. Status \& Scope Backhuys Publishing. Amsterdan, Netherlands. Pp. 135-149.

Montejano, G., M. Gold-Morgan y H. León-Tejera. 2005. Surveying the diversity of Cyanoprokaryotes in poorly known regions: the case of the central region of México. Algological Studies 117(1): 329-338. DOI: https://doi.org/10.1127/18641318/2005/0117-0329

Montejano, G., M. Gold-Morgan y I. Becerra-Absalón. 2017. Nematoplaca incrustans Geitler (Nostocales, Cyanobacteria/Cyanoprokaryota): reinterpretation of the life cycle and validation of the generic status. Nova Hedwigia 105(1-2): 29-36. DOI: https://doi.org/10.1127/ nova_hedwigia/2017/0396

Montejano, Z. G., I. Becerra-Absalón, M. Gold-Morgan y K. Osorio. 2018. Gloeobacter violaceus: primitive reproductive scheme and its significance. Plant Systematics and Evolution 304(10): 1221-1229. DOI: https://doi.org/10.1007/s00606018-1542-z

Montoya, M. Y. y R. N. Aguirre. 2013. Estado del arte del conocimiento sobre perifiton en Colombia. Gestión y Ambiente 16(3): 91-117. DOI: https://doi.org/10.15446/ga

NCBI. 2020. Phormidium. National Center for Biotechnology Information, NCBI Resources. Bethesda, USA. http://www. ncbi.nlm.nih.gov/nuccore/?term=Phormidium (consultado en enero de 2020).

Necchi, O. Jr., C. C. Z. Branco, R. C. G. Simão y L. H. Z. Branco. 1995. Distribution of stream macroalgae in the northwest region of São Paulo State, southeastern Brazil. Hydrobiologia 299: 219-230. DOI: https://doi.org/10.1007/BF00767329

Ortega Murillo, M., R. Hernández, O. Vázquez, R. Alvarado y M. Martínez. 2015. La ficoflora de un lago monomíctico en Michoacán, México. Cymbella 1: 26-45.

Parker, T. C., J. B. Tindall y M. G. Garrity. 2019. International Code of Nomenclature of Prokaryotes. International Journal of Systematic and Evolutionary Microbiology 69(1A): 1-111. DOI: https://doi.org/10.1099/ijsem.0.000778

Perona, E., I. Bonilla y P. Mateo. 1998. Epilithic cyanobacterial communities and water quality: an alternative tool for monitoring eutrophication in the Alberche River (Spain). Journal of Applied Phycology 10: 183-191. DOI: https://doi. org/10.1023/A:1008051327689 
Potts, M. 1999. Mechanisms of desiccation tolerance in cyanobacteria. European Journal of Phycology 34(4): 319328. DOI: http://dx.doi.org/10.1080/09670269910001736 382

Ramírez, M., M. Hernández-Mariné, P. Mateo, E. Berrendero y M. Roldán. 2011. Polyphasic approach and adaptative strategies of Nostoc cf. commune (Nostocales, Nostocaceae) growing on Mayan monuments. Fottea 11(1): 73-86.

Rodarte, B., I. Becerra-Absalón, G. Montejano, K. Osorio, H. León y C. Segal. 2014. Morphological and Molecular Characterization of Brasilonema roberti-lamii (Cyanophyceae, Nostocales, Scytonemataceae) from Central Mexico. Phytotaxa 164(4): 255-264. DOI: https:// dx.doi.org/10.11646/phytotaxa.164.4.4

Rodríguez-Flores, R. y J. Carmona. 2018. Ecology and distribution of macroscopic algae communities in streams from the Basin of Mexico. Botanical Sciences 96(1): 63-75. DOI: http://doi.org/10.17129/botsci.1237

Scott, J. T. y A. M. Marcarelli. 2012. Cyanobacteria in freshwater benthic environments. In: Whitton, B. (ed.). Ecology of Cyanobacteria II. Their Diversity in Space and Time. Springer. Dordrecht, Netherlands. Pp. 271-289. DOI: https://doi. org/10.1007/978-94-007-3855-3_9

Segura, G. M. 2018. Análisis polifacético de las especies de Microcystis (Cyanoprokaryota, Chroococcales) en tres lagos urbanos del centro de México. Maestría en Ciencias Biológicas. Facultad de Ciencias, Universidad Nacional Autónoma de México. Cd. Mx., México. 108 pp.

Sheath, R. G. y K. M. Cole. 1992. Biogeography of stream macroalgae in North America. Journal of Phycology 28(4): 448-460. DOI: https://doi.org/10.1111/j.00223646.1992.00448.x
Tonetto, F. A., K. C. Peres, R. T. Hirata y B. C. Zanini. 2015. Spatial and temporal distribution of stream macroalae in a tropical river basin. Biota Neotropica 15(4): e0095. DOI: http:// dx.doi.org/10.1590/1676-0611-BN-2015-0095

Trejo, C. 2018. Caracterización morfológica y molecular de especies de cianobacterias de la familia Rivulariaceae encontradas en dos lagos-cráter del estado de Puebla, México. Tesis de licenciatura. Facultad de Ciencias, Universidad Nacional Autónoma de México. Cd. Mx., México. 102 pp.

Turland, N. J., J. H. Wiersema, F. R. Barrie, W. Greuter, D. L. Haeksworth, P. S. Herendeen y T. W. May. 2018. International Code of Nomenclature for algae, fungi and plants (Shenzhen Code) adopted by the Nineteenth International Botanical Congress Shenzhen, China, July 2017. Koeltz Botanical Books. Glashütten, Germany. DOI: https://doi. org/10.12705/Code.2018

Valadez-Cruz, F., J. Carmona y E. Cantoral. 1996. Algas de ambientes lóticos en el estado de Morelos, México. Anales del Instituto de Biología, Universidad Nacional Autónoma de México 67(2): 227-282.

Zanini, B. C., A. R. Krupek y C. K. Peres. 2009. Distribution of stream macroalgal communities from the mid-western region of Paraná state, southern Brazil: importance of local scale variation. Brazilian Archives of Biology and Technology 52(2): 379-386. DOI: http://dx.doi.org/10.1590/S151689132009000200015 